\title{
Monte Carlo Simulation for the Formation and Growth of Low Dimensionality Phases During Underpotential Deposition of $\mathrm{Ag}$ on $\mathrm{Au}(100)$
}

\author{
M.C. Giménez, M.G. Del Pópolo and E. P. M. Leiva *
}

October 26, 2018

\begin{abstract}
Simulation studies are undertaken for the system $\mathrm{Ag} / \mathrm{Au}(100)$ by means of grand canonical Monte Carlo applied to a large lattice system. The interactions are calculated using the embedded atom model. The formation of adsorbed $\mathrm{Ag}$ phases of low dimensionality on $\mathrm{Ag}(100)$ is investigated and the influence of surface defects on the shape of the adsorption isotherms is studied. The results of the simulations are discussed in the light of experimental information available from electrochemical measurements.
\end{abstract}

Keywords: underpotential deposition, embedded atom method, Monte Carlo simulation.

\section{Introduction}

New horizons have opened concerning the understanding of metal electrodeposition processes in the last decade. This has been achieved to a large extent due to the use of electrochemical techniques like cyclic voltammetry along with in-situ nanoscopy techniques. Among these, scanning tunnelling microscopy (STM) has allowed the observation in direct space and real time of the formation and growth of a new phase during the electrocrystallization of a metal from an electrolytic solution. In this context we can mention the so-called underpotential deposition (upd), which implies the deposition of a metal $M$ on the surface of an electrode or substrate $S$ of different nature at potentials more positive than those predicted from the Nernst equation. A recent and extensive discussion on this phenomenon can be found in a book of Budevski, Staikov and Lorenz 1, and the theoretical aspects have been considered in reviews by one of us 2, 3.

One way to quantify upd is through the so-called underpotential shift $\triangle \phi_{U P D}$, which is related to the difference of the chemical potential of $M$ adsorbed on $S$ at a coverage degree $\Theta$, say $\mu\left(M_{\Theta} / S\right)$, and the chemical potential of $M$ in the bulk phase, say $\mu(M / M)$, through the following equation:

$$
\Delta \phi_{U P D}=\frac{1}{z e_{0}}\left[\mu(M / M)-\mu\left(M_{\Theta} / S\right)\right]
$$

\footnotetext{
${ }^{*}$ Corrresponding autor: E. P. M. Leiva. e-mail:eleiva@mail.fcq.unc.edu.ar
} 
where $z$ is the charge of the ion $M$ in the solution and $e_{0}$ is the elemental charge. Criteria for the experimental estimation of $\Delta \phi_{U P D}$ have been given in the literature by Kolb et al. 4] and Trasatti [5]. Concerning the modelling of upd as a thermodynamic process, different interesting approaches have been taken by several authors in the 70's $1,6,6,8,9$. The most widespread idea is to envisage this phenomenon as related to the formation of different adsorbed phases, involving in some cases, phases of low dimensionality that differ in their properties and can be detected as separate entities by a given experimental techniques, i.e. peaks in a voltammogram. The interpretation of upd as a phase transition has been employed by Blum et al 9 to develop a model for the underpotential deposition of $\mathrm{Cu}$ on $\mathrm{Ag}(111)$ in the presence of sulfate anions and has also been recently brought to a more general context by Lorenz and coworkers [10]. According to these ideas, the dimensionality of the phases involved in the upd phenomena is between 0 and 2. The $0 \mathrm{D}$ phases correspond to the adsorption of isolated metal atoms, the adsorption on kink sites, the adsorption on vacancies of the first lattice plane of the substrate or on some other type of point defects. The 1D phases are related to the adsorption at the border of steps or monoatomic terraces and the $2 \mathrm{D}$ phases are related to the formation of monolayers and submonolayers of adsorbate building compressed, (1x1) or even expanded structures.

Figure 1 illustrates the most common situations experimentally observed, which will also be considered in the present simulations studies.

It can be observed that relative abundance of $0 \mathrm{D}$ and $1 \mathrm{D}$ phases is determined by the presence of defects on the electrode surface. Its existence has been related to the existence of small shoulders in the voltammograms, as postulated by Siegentaler et al. 11 and Lorenz and coworkers 12. In the case of the $2 \mathrm{D}$ phases that are formed on the flat terraces or defect-free surfaces there is clear experimental evidence for the existence of compressed [13, compact and expanded structures [1, 10. The formation and growth of a compact, defectfree bi-dimensional structure can be described as a first-order phase transition that takes place at the electrode/solution interface and its occurrence should be characterized by a discontinuity in the adsorption isotherm, with a concomitant very sharp peak (more properly speaking a Dirac delta function) in the voltammetric profiles [9]. Concerning this point, it is remarkable that although upd has been often related to the presence of first-order phase transitions, the real existence of these phenomena in experimental systems is still not completely free of controversy, since discontinuities in the experimental isotherms are usually not obtained when specific anion adsorption is avoided [1]. We arrive thus at the somewhat paradoxical situation where experimentalists usually perform their experiments under conditions where the clean adsorption process of metal adsorption is actually coupled to the even more complex process of anion coadsorption. Higher order phase transitions should be characterized by the presence of rather wide and flat voltammetric peaks 9 , though there is no experimental evidence for the occurrence of this type of phenomena in electrochemical systems [1]. In this respect, computer simulations could also deliver enlightening information. Furthermore, computer simulations play a fundamental role for the comprehension of surface phenomena 21. Many approaches can be taken for the study of surface-related problems. Among others, we can mention molecular 
dynamics simulations, Monte Carlo simulations based on continuum Hamiltonians (off-lattice) and techniques in which the surface is modelled through a lattice Hamiltonian (on-lattice simulations) [22].

The present work deals with the computer simulation of $\mathrm{Ag}$ deposition of $\mathrm{Au}(100)$ by means of the Grand Canonical Monte Carlo method and the interatomic potential given by the embedded atom method (EAM), which has been previously employed by some of us to study electrochemical systems [14. We developed a simulation scheme that combines the simplicity of the lattice model of the surface with the many body properties of the metallic binding. Adsorption isotherms under different temperature conditions were obtained in order to analyze the existence of a first-order phase transition, and the influence of different surface defects on the behaviour of the isotherms is also investigated.

\section{Some experimental facts}

Cyclic voltammetry and STM in-situ experiments performed on the systems $\mathrm{Ag} / \mathrm{Au}(\mathrm{hkl})$ have shown the presence of different adsorbed phases, depending on the applied electrode potential [10, 18, 19, 20. In the particular case of $\mathrm{Ag}$ adsorption on $\mathrm{Au}(100)$, the experiments have revealed the existence of an expanded, quasi hexagonal $c(\sqrt{2} \times 5 \sqrt{2}) R 45^{\circ} \mathrm{Ag}$ phase at high overpotentials. The existence of this expanded structures can hardly be explained in terms of energetic considerations, so that the entropic contributions to the free energy of the system should be considered. Furthermore, the presence of specifically adsorbed anions may in turn play an important role in stabilizing this type of entities [16, so we shall leave the consideration of this type of structures for future work. On the other hand, at intermediate underpotentials the decoration of monoatomic steps corresponding to the formation of a one-dimensional phase was observed, while at underpotentials close to Ag bulk deposition, two dimensional islands were built on terraces and defect-free zones of the substrate. STM measurements with lateral atomic resolution showed that these islands present a quadratic symmetry, suggesting that they may be considered as a $(1 \times 1)$ structure commensurate with the $\mathrm{Au}(100)$ substrate. Finally, it must be added that long time polarizations showed some indication for the beginning of a surface alloying process whose mechanism has so far not been explained.

\section{Model and simulation method}

\subsection{Lattice model}

Lattice models for computer simulations are of widespread use in studies of nucleation and growth, because they allow dealing with a large number of particles at a relatively low computational cost. In principle, it must be kept in mind that continuum Hamiltonians should be much more realistic in those cases where epitaxial growth of an adsorbate leads to incommensurate adsorbed phases [23] or to adsorbates with large coincidence cells. On the other hand, the use of fixed rigid lattices restricts enormously the number of possible configurations for the adsorbate and its use may be justified on the basis of experimental evidence or continuum computer simulations that predict a proper fixed lattice geometry. In the present case, we have strong evidence from this latter type of simulations 
within the canonical Monte Carlo method 24 that indicates that at least one of the phases present during $\mathrm{Ag}$ underpotential deposition on $\mathrm{Au}(100)$ possesses a pseudomorphic structure. In fact, our continuum MC simulations showed that a $\mathrm{Ag}$ monolayer adsorbed on $\mathrm{Au}(100)$ spontaneously acquired a $(1 \times 1)$ coincidence cell in agreement with the experimental finding at low underpotentials already mentioned in section 2 . For this reason, we shall employ here a lattice model to represent the square (100) surface lattice in a Grand Canonical Monte Carlo simulation. Besides Ag adatoms, Au adatoms are allowed on the surface at different coverage degrees, building a variety of surface structures with the purpose of emulating some of the most common surface defects. Linear sweeps of the chemical potential are performed to obtain the adsorption isotherms and study the influence of the surface defects on their shapes. First of all, we neglect the effect of the presence of solvent molecules. This approximation should not be critical as long as the partial charge on the adatoms is small, thus minimizing the ion-dipole interactions. Second, we also neglect all kinds of anion effects that may coadsorb during the metal deposition process. In the case of other systems, the adatom-anion interactions have been shown to be very important [17, playing a decisive role in determining the energetics of the system. This has been recently analyzed by some of us in thermodynamic terms [16. In the case of the present system, however, thin layer twin electrode experiments gave no indication for any change in the extent of anion adsorption upon building the adsorbed monolayer.

Square lattices of different sizes with periodical boundary conditions are used in the present work to represent the surface of the electrode. Each lattice node represents an adsorption site for a $\mathrm{Ag}$ or a $\mathrm{Au}$ atom. The former may adsorb, desorb or jump between neighboring sites, while the latter may only move on the surface like the Ag atoms do. In this way, our model corresponds to an open system for one of its components, that is, Ag. This has physical correspondence with the setup of the electrochemical experiment, where only the metal in equilibrium with its cations in solution may dissolve or be deposited in the potential range considered.

Concerning the $\mathrm{Au}$ adatoms, some considerations must be made regarding the existence of surface defects. These atoms may in principle move freely on the surface and minimize the free energy of the system by a number of mechanisms. For example, isolated $\mathrm{Au}$ atoms may heal defects through their incorporation to a defective cluster, or small $\mathrm{Au}$ islands may dissolve to join large ones, like shown in previous simulation work by Stimming and Schmickler [25]. However, as long as these processes are slow enough as compared with the processes related to the $\mathrm{Ag}$ adatoms (and this is found to be the case), the whole process can be envisaged as the adsorption/desorption behaviour of $\mathrm{Ag}$ on a $\mathrm{Au}(100)$ surface with defects. Thus, different Au structures can be imposed as initial conditions for each simulation. In the present case, they range from islands of different sizes and shapes obtained by means of simulated annealing techniques, up to completely random distributions. All this was undertaken in order to emulate some of the defects that can be found on a real single crystal surface, like kink sites, vacancies, isolated substrate atoms, steps, etc. 


\subsection{Interatomic potential}

A very important feature to be taken into account when comparing the results of a simulation with experiment is the quality of the interatomic potentials used to perform the simulations. Several methods have been developed to calculate the total energy of a many-particles metallic system, with a computational effort comparable to that of a pair potential 26. Among these models, denominated broken-bonds or bond-cutting methods it is worth mentioning the embedded atom method(EAM) [27, the N-body potentials of Finnis and Sinclair 28, the second-moment approximation or Tight-binding (TB) [26] and the glue model (GM) 29]. In this work we use the embedded atom method 27] because it is able to reproduce important characteristics of the metallic binding that cannot be obtained using simple pair potentials.

The EAM considers that the total energy $U_{\text {tot }}$ of an arrangement of $N$ particles may be calculated as the sum of energies $U_{i}$ corresponding to individual particles

$$
U_{t o t}=\sum_{i=1}^{N} U_{i}
$$

where $U_{i}$ is given by

$$
U_{i}=F_{i}\left(\rho_{h, i}\right)+\frac{1}{2} \sum_{j \neq i} V_{i j}\left(r_{i j}\right)
$$

$F_{i}$ is denominated embedding function and represents the energy necessary to embed atom $i$ in the electronic density $\rho_{h, i}$ at the site at which this atom is located. $\rho_{h, i}$ is calculated as the superposition of the individual electronic densities $\rho_{i}\left(r_{i j}\right)$ :

$$
\rho_{h, i}=\sum_{j \neq i} \rho_{i}\left(r_{i j}\right)
$$

Thus, the attractive contribution to the EAM potential is given by the embedding energy, which accounts for many-body effects. On the other hand, the repulsion between ion cores is represented through a pair potential $V_{i j}\left(r_{i j}\right)$, which only depends on the distance between the cores $r_{i j}$ :

$$
V_{i j}=\frac{Z_{i}\left(r_{i j}\right) Z_{j}\left(r_{i j}\right)}{r_{i j}}
$$

$Z_{i}\left(r_{i j}\right)$ may be envisaged as a sort of effective charge, dependent on the nature of the particle $i$. The EAM has been parametrized to fit experimental data like elastic constants, dissolution enthalpies of binary alloys, bulk lattice constants and sublimation heaths 27. Pair functionals have been widely used for surface diffusion studies and adsorption of metals on metallic surfaces [14, 30, 31.

\subsection{Grand Canonical Monte Carlo}

One of the most appealing characteristics of Grand Canonical Monte Carlo $(\mu V T / M C)$ is that the chemical potential $\mu$ is one of the independent variables, as it is the case in many experimental situations. In the electrochemical case, we can mention low-sweep rate voltammetry, where the electrode potential can be used to control the chemical potential of species at the metal/solution interface. This technique offers a straightforward way of obtaining the adsorption 
isotherms provided the sweep rate is low enough to ensure equilibrium for the particular system considered. Another interesting aspect of $\mu V T / M C$ is that a better and faster equilibration of the system is possible due to local density changes originated by fluctuations in the number of particles.

Our 2D system is characterized by a square lattice with $M$ adsorption sites. We shall label each adsorption site with a number 0,1 or 2 , depending on whether it is empty or occupied by a gold or a silver atom respectively. Thus, the configuration of the system will be defined through the components $n_{1}$, $n_{2}, \cdots, n_{M}$ of an $M$-dimensional vector $\vec{n}$ and its partition function may be written as:

$$
\Xi\left(\mu_{1}, \mu_{2}, M, T\right)=\sum_{\vec{n}} \exp \beta\left(\mu_{1} \sum_{i=0}^{M} \delta_{1, n_{i}}+\mu_{2} \sum_{i=0}^{M} \delta_{2, n_{i}}\right) \exp (-\beta U(\vec{n}))
$$

where $\mu_{1}$ and $\mu_{2}$ are the chemical potentials of gold and silver at the interface, $\delta_{1, n_{i}}$ and $\delta_{2, n_{i}}$ are Kronecker delta functions acting on the components of the vector $\vec{n}$ and $U(\vec{n})$ is the energy of the system in state $\vec{n}$. The probability of obtaining a given configuration will be given by the corresponding Boltzmann factor:

$$
P_{\vec{n}}\left(\mu_{1}, \mu_{2}, M, T\right)=\frac{\exp \beta\left(\mu_{1} \sum_{i=0}^{M} \delta_{1, n_{i}}+\mu_{2} \sum_{i=0}^{M} \delta_{2, n_{i}}\right) \exp (-\beta U(\vec{n}))}{\Xi\left(\mu_{1}, \mu_{2}, M, T\right)}
$$

Following the procedure proposed by Metropolis and coworkers [32, the acceptance probability for a transition from state $\vec{n}$ to $\vec{n}^{\prime}$ is defined as:

$$
W_{\vec{n} \rightarrow \vec{n}^{\prime}}=\min \left(1, \frac{P_{\vec{n}^{\prime}}}{P_{\vec{n}}}\right)
$$

so that detailed balance is granted.

In our $\mu V T$ / $M C$ simulation we shall allow for three types of events :

1. Adsorption of a Ag atom on a lattice site selected randomly. According to eqns, 7 and 8 , the acceptance probability $W \vec{n} \rightarrow \vec{n} \vec{n}^{\prime}$ for this event is:

$$
W_{\vec{n} \rightarrow \vec{n}^{\prime}}=\min \left(1, \exp \left(\beta\left(\mu_{1}-\Delta U\right)\right)\right)
$$

where $\Delta U=U\left(\vec{n}^{\prime}\right)-U(\vec{n})$.

2. Desorption of a $\mathrm{Ag}$ atom selected randomly. This probability is

$$
W_{\vec{n}} \rightarrow \vec{n}^{\prime}=\min \left(1, \exp \left(\beta\left(-\mu_{1}-\Delta U\right)\right)\right)
$$

3. Motion of a $\mathrm{Ag}$ or a $\mathrm{Au}$ atom from the lattice site where it is adsorbed to one of its four nearest neighbors. The latter is selected randomly and the acceptance probability for this event is equivalent to that employed in the canonical ensemble, since the number of particles remains constant. We have:

$$
W_{\vec{n} \rightarrow \vec{n}^{\prime}}=\min (1, \exp (-\beta \Delta U))
$$


Even when in a grand canonical simulation, events of type 3 (motion or diffusion of lattice particles) are not strictly necessary, their presence is justified by the fact that a smaller number of Monte Carlo steps(MCS) must be employed for the equilibration of the system 32 . The calculated equilibrium thermodynamic properties do remain unaltered by this choice.

In order to satisfy detailed balance, the a priori probability for the creation of a $\mathrm{Ag}$ atom, $\alpha_{c}$, must be equal to the probability of its anhilation $\alpha_{d}$ and independent of its motion probability $\alpha_{m}$.

The algorithm devised for an elementary step of the simulation is the following:

1. Selection of the elementary process(adsorption, desorption of diffusion) to occur. This is made by means of the generation of a random number $\epsilon$ uniformly distributed in the interval $[0,1]$.

2. If $0 \leq \epsilon \leq\left(\alpha_{c}+\alpha_{d}\right)$, a random site is chosen on the lattice.

- If the occupation number of the site is 0 , an attempt is made to create a $\mathrm{Ag}$ atom at that site, according to the acceptance given by eqn. 9 .

- If the occupation number of the site is 2 , an attempt is made to destroy the atom adsorbed at that site, according to the acceptance given by eqn. 10 .

3. If $\left(\alpha_{c}+\alpha_{d}\right) \leq \epsilon \leq 1$, an attempt is made to move each of the particles of the system according to the following algorithm:

- One of the nearest sites to the particle is selected. If the occupation number is 0 , a movement to the new position is attempted according to eqn. 11. Otherwise, the next particle in the list is considered.

4. Back to point 1 , thus completing one MCS.

Within this procedure, the relevant thermodynamic properties are then obtained as average values of instantaneous magnitudes stored along a simulation run. For example, the average coverage degree of the silver atoms $\langle\Theta\rangle_{A g}$ at a given chemical potential $\mu_{1}$ after a simulation with $M C S_{e}$ equilibration steps and a total number of $M C S$ steps will be given by:

$$
\left\langle\Theta\left(\mu_{1}\right)\right\rangle_{A g}=\frac{1}{M C S-M C S_{e}} \sum_{i=M C S_{e}}^{M C S} \Theta\left(\mu_{1}\right)_{A g, i}
$$

where the instantaneous value of silver coverage degree, $\Theta\left(\mu_{1}\right)_{A g, i}$, is defined from the number of $\mathrm{Ag}$ atoms $N_{A g, i}$ and the number of $\mathrm{Au}$ atoms $N_{A u}$ present at the interface at the time step $i$

$$
\Theta\left(\mu_{1}\right)_{A g, i}=\frac{N_{A g, i}}{M-N_{A u}}
$$

Since our main goal is to obtain adsorption isotherms for several Ag coverage degrees and different surface structures of $\mathrm{Au}$, we chose a simulation method that does not deliver information on the kinetics of nucleation and growth. In this respect, the Kinetic Monte Carlo(KMC) 33, 34 technique should be applied and will be the subject of future work. 


\subsubsection{Algorithm employed for the calculation of energy differences}

In a $\mathrm{MC}$ simulation the determining step concerning the speed of the calculation is the evaluation of the total energy of the system $U(\vec{n})$, which is determined by the type of interatomic potential employed.

According to the algorithm described in the previous section, any new state of the system is accepted with a probability that involves the Boltzmann factor of the energy difference between the initial and the final state, that is $\Delta U=U\left(\vec{n}^{\prime}\right)-U(\vec{n})$. One of the main advantages of the lattice model is its simplicity, since it fixes the distances between the adsorption nodes, thus reducing the energy values that the system can take to a discrete set. Furthermore, the potentials used are short ranged, so that a very important simplifying assumption can be made for obtaining $\Delta U$. The point is to consider the adsorption(desorption) of a particle at a node immersed in a certain environment surrounding it, as shown in Figure 2 . The adsorption site for the particle is located in the central box, and the calculation of the interactions is limited to a circle of radius $R$. Then, the adsorption energy for all the possible configurations of the environment of the central atom can be calculated previous to the simulation. The $S$ adsorption sites within the circle are labelled arbitrarily with numbers between 1 and $S$ (Figure 2) and their labels along with the occupation number may be employed to identify the configuration in terms of a number $I$ expressed on the basis of 3 . Generally speaking, for a configuration characterized by the occupation numbers $n_{1}, n_{2}, \ldots n_{S}$, the corresponding value of $I$ is calculated as $I=\sum_{i=1}^{S-1} n_{i} 3^{S-i-1}$. For example, for an environment with 8 neighbors $(S=9)$ such as that shown in Figure 2a, the configuration $n_{1}=2, n_{2}=2, n_{3}=1, n_{4}=1, n_{5}=0, n_{6}=0, n_{7}=0, n_{8}=0$ is indexed according to our convention with $I=6156$. With this method all the adsorption/desorption energies of $\mathrm{Ag}$ and $\mathrm{Au}$ on $\mathrm{Au}(100)$ are tabulated, so that during the $\mathrm{MC}$ simulation the most expensive numerical operations are reduced to the reconstruction of the number $I$ that characterizes the configuration surrounding the particle on the adsorption node. Computationally speaking, $I$ is nothing but the index of the array in which the energy is stored.

In the present work, the adsorption/desorption energies were calculated for environments with $R$ equal to the distance between second and third nearest neighbors, as shown in Figure 2 , using as a substrate a five-atomic planes thick gold slab. The results for the energy are shown as a histogram in Figure 3 , where the frequency of appearance of a given energy is shown as a function of the adsorption energy. This is a very important information, since this figure contains the distribution of energy changes that an atom may undergo during the simulation. A more detailed discussion on the information that can be extracted from the histograms will be given in section 4.1.

We must finally mention a further assumption in our calculations. In all the structures generated the adsorbates were located at a vertical distance from the first substrate plane equivalent to the distance between neighboring $\mathrm{Au}(100)$ lattice planes. This should not be a very important approximation, since the difference between the $\mathrm{Au}$ and $\mathrm{Ag}$ lattice constants amounts only $0.01 \AA$. 


\subsubsection{Simulated annealing}

Simulated annealing techniques have often been used to obtain minimal energy structures or to solve ergodicity problems. A suitable way to implement them is through the canonical Monte Carlo method at different temperatures. The simulation is started at a very high initial temperature $T_{o}$, of the order of $10^{5}$ $\mathrm{K}$, and the system is later cooled down following a logarithmic law:

$$
T_{f}=T_{o} K^{N_{\text {cycles }}}
$$

where $\mathrm{T}_{f}$ is the final temperature, $N_{\text {cycles }}$ is the number of cooling steps and $K$ is a positive constant lower than one. A few hundreds of thousands of MCS are run at each temperature in order to allow an extensive exploration of the configuration space and the simulation stops when $T_{f}$ is reached. Although experimental annealing treatments (like flame annealing) are used to get clean and structurally well defined surfaces, in the present case we have used the computational homologue to obtain the more stable surface defects of Au at $300 \mathrm{~K}$. In the case of experiments, this procedure has been sometimes questioned and we will see here that the type of structures obtained are indeed very sensitive to the cooling rate employed.

\section{Results and discussion}

\subsection{Some considerations on the adsorption/desorption en- ergies of Ag}

The information provided by the histograms shown in Figure 3 is very valuable since they contain all the possible adsorption/desorption energies that a Ag atom may undergo during the simulation. For the purpose of the present analysis, we roughly distinguish between three types of configurations: those in which the central atom (box 13 in fig. $2 \mathrm{~b}$ ) is surrounded only by other Ag atoms(or empty sites), those in which the central atom is only surrounded by Au atoms and those in which the environment may contain $\mathrm{Au}$ or $\mathrm{Ag}$ atoms indistinctly. The two former families involve 4096 configurations each, while the latter consists of 531441 configurations, including the two previous ones. In can be observed that in the two former cases (continuous and dotted histograms respectively) the energy distribution is multimodal, they being qualitatively very similar to each other. However, the histogram corresponding to Ag adsorption in the presence of $\mathrm{Au}$ atoms is flatter and is shifted towards more negative adsorption energies. This suggests a certain affinity of $\mathrm{Ag}$ for a $\mathrm{Au}$ environment. From this point of view, the adsorption of $\mathrm{Ag}$ in the presence of $\mathrm{Au}$ surface defects should be favoured with respect to the adsorption on a perfect surface. This speculation will be corroborated by the MC simulations.

The pulse-plot in Figure 3 corresponds to the distribution of the adsorption energies in the mixed environment for the 12-neighbors system. The energy distribution looks still multimodal but considerably smoother than the previous ones. Thus, in general terms it can be concluded that the presence of gold atoms surrounding the adsorbed $\mathrm{Ag}$ atoms as surface defects will widen and enrich the energy spectrum of the adsorbate. 


\subsection{Ag deposition on a perfect $\mathrm{Ag}(100)$ single crystal sur- face.}

Our initial work was devoted to the deposition of $\mathrm{Ag}$ on a defect-free $\mathrm{Au}(100)$ surface, performing $\mu V T / M C$ simulations at $300 \mathrm{~K}$. The systems consisted in $(40 \times 40)$ and $(100 \times 100)$ lattices, using the adsorption/desorption energy tables calculated as described in Section 3.3.1.

Positive and negative chemical potential sweeps were made in the range between $-2.7 \mathrm{eV}$ and $-3.2 \mathrm{eV}$, involving simulations with a length of $10^{6}$ to $10^{7} M C S$, depending on the system size. In order to quantify underpotential deposition, the deposition of $\mathrm{Ag}$ on $\mathrm{Ag}(100)$ was also simulated. The chemical potential for bulk Ag deposition $\mu_{A g / A g}^{c}$ was determined from these runs.

In all cases the adsorption isotherms show a very abrupt change of the coverage degree, Fig. 4a, at a characteristic chemical potential $\mu_{A g / A u}^{c}$, that mainly depends on the cutoff radius employed for the calculation of the energy and to a lower extent on the system size. The $\mu_{A g / A u}^{c}$ values along with other relevant information are reported in Table 1 . The drastic change observed in the adsorption isotherms points towards the existence of a first-order phase transition in which a two-dimensional phase (an adsorbed monolayer) is built through a nucleation and growth process. According to the values of chemical potentials reported in Table 1, this occurs at lower $\mu$ values than those expected for bulk Ag deposition $\left(\mu_{A g / A g}^{c}=-2.865 \mathrm{eV}\right.$ ), leading to an underpotential shift $\Delta \phi_{U P D}=\mu_{A g / A g}-\mu_{A g / A u}$ that can be estimated to be between $0.06 \mathrm{eV}$ and $0.11 \mathrm{eV}$. The isotherm corresponding to $\mathrm{Ag}$ adsorption on $\mathrm{Ag}(100)$ is also shown in Figure 4a.

Concerning the chemical potential sweep, it was performed in two different fashions. In the first, for each new $\mu$ value characterizing the simulation, an initial value of coverage degree of $\mathrm{Ag}, \Theta_{A g}$, was set, that was $0,0.5$ or 1 . This delivered three types of isotherms that corresponded to the different initial conditions. In the second type of simulations, the final configuration at a given $\mu$ was employed as starting point for the next simulation. The first type of simulations was employed to study hysteresis effects, that in our system were found to be important at $300 \mathrm{~K}$ (Figure 4b). As shown below, they are related to the absence of surface defects since it is precisely on these defects where the formation of the new phase starts. The simulations undertaken with the second scheme showed essentially the same characteristics as those with the first.

The effect of temperature was also investigated and it was found that at high temperatures the sharp behaviour of the $\Theta-\mu$ isotherms changes to a smooth, langmuirian-like behavior, as can be seen in Figure 5. From these curves we can infer that at $300 \mathrm{~K}$ the present system is well below its critical temperature. We can also estimate this critical temperature using the equation of the 2-D Ising model:

$$
T_{c}=\frac{w}{2 k \ln (\sqrt{2}-1)}
$$

where $k$ is the Botzmann constant and $w$ is the interaction energy between nearest neighbours. We have estimated a value of $w$ by inserting a $\mathrm{Ag}$ adatom in different environments. The values obtained were in the range $-0.27 \mathrm{eV}<$ $w<-0.18 \mathrm{eV}$ so that the critical temperature is estimated to be in the range 
$1200 \mathrm{~K}<T_{c}<1800 \mathrm{~K}$. These values should only provide a rough estimation, since the Ising model does not consider the many body effects present here.

\subsection{Influence of surface defects on the adsorption isotherms}

A wide variety of defects may exist on a single crystal surface, to an extent that it depends among other factors on the temperature, the chemical environment and the method by which it was generated. We therefore performed an important number of simulations to elucidate the effect of the most common surface defects on the Ag adsorption isotherm. The starting configuration for the Au defects on the surface was obtained from a simulated annealing calculation and a $100 \times 100$ lattice and $R=3$ were used in all cases. The length of the simulations ranged between $10^{6}$ and $10^{7} M C S$, with an adequate number of equilibration steps.

\subsubsection{Generation of surface inhomogeneities by means of simulated annealing}

It has been demonstrated experimentally that after a flame annealing treatment the $\mathrm{Au}(100)$ surface is reconstructed to give a quasi-hexagonal structure 35. This reconstruction is lifted upon a potential sweep at a potential value that depends on the nature of the electrolyte involved. Since the hexagonal structure is more compact than the $(1 \times 1)$ structure, a number of islands are built on the electrode surface that accommodate the remaining atoms. In the case of the experimental studies of Ag deposition on $\mathrm{Au}(100)$, STM and AFM in-situ images indicate that previous to Ag deposition the substrate consists in terraces separated by monoatomic steps, while the corresponding images with lateral atomic resolution show a quadratic structure characteristic for an unreconstructed surface 10, 12].

Since the atomic excess expected after the lifting of the reconstruction is about $13 \%$ of the atomic density of the $\mathrm{Au}(100)$ single crystal surface, we generated $\mathrm{Au}$ islands that cover the single crystal surface with a coverage degree $\Theta_{A u} \simeq 0.1$. Thus, simulated annealing was performed with $1.000 \mathrm{Au}$ atoms distributed on a $100 \times 100$ lattice at an initial temperature $T_{0}$ of $10.000 \mathrm{~K}$. The system was later cooled down to a final temperature $T_{f}=300 \mathrm{~K}$ following the cooling rate given by eqn. 14 with $K=0.8$. This produced 16 cooling steps between the initial and the final temperature. In order to produce surfaces with different densities of defects, the number of Monte Carlo steps allowed at each temperature was varied, obtaining the different final structures shown in Figure 6 . It can be noticed that when the number of $M C S$ at each temperature is increased, the islands turn more compact, with well defined borders. This is equivalent to a low concentration of defects such as kinks and monoatomic steps. On the other hand, if the cooling is faster, the number of islands per unit surface increases and their borders turn more irregular. Thus, the lower the number of $M C S$ allowed at each temperature, the farther the final state will be from equilibration. Table 2 shows the density of kink sites $\rho_{k}$ and the density of sites located at the border of the monoatomic steps $\rho_{s}$ at different number of $M C S$. The $\rho_{k}$ was calculated as $\frac{M_{k}}{M}$ where $M_{k}$ is the number of kink sites and $M=N^{2}$ is the total number of lattice sites(10.000). Analogously, $\rho_{s}$ was calculated as $\frac{M_{s}}{M}$ where $M_{s}$ is the number of sites located at the border of the monoatomic steps. The relationship perimeter/area of the islands, calculated 
from the number of $\mathrm{Au}$ atoms located at the border and the number of atoms located inside the islands, is also given in the table. All the values refer to the final configuration of the system.

\subsubsection{Deposition on kink sites}

The first stage in the deposition process is given by the adsorption of $\mathrm{Ag}$ atoms on point defects. This can be envisaged as the formation of a 0-dimensional phase that occurs at chemical potentials more negative than the chemical potential for the formation of the Ag monolayer. Although the most commonly observed point defects on the surface of a monocrystalline electrode at $300 \mathrm{~K}$ are kink sites, their density at this temperature may be affected by different factors, like the presence of a condensed phase in contact with the metallic surface 11. In the present work we considered that the initial number of kink sites and the corresponding roughness of the monoatomic terraces of the substrate only depends on the cooling rate employed in the simulated annealing process. The initial structure of the stepped Au surface was set according to the results obtained in section 4.3.1 and sweeps of chemical potential were undertaken between -3.30 and $-3.02 \mathrm{eV}$. Figure $7 \mathrm{a}$ shows isotherms obtained with 5 of the 16 configurations resulting from the simulated annealing process. The gold structures considered correspond to the final state resulting from simulations with $20,320,5120,81920$ and $655360 M C S$ and range from polyhedron shaped islands with different roughness up to a more or less random distribution of very small irregular islands. A coverage degree due to adsorption on kink sites $\Theta_{k}$ may be defined considering that they form a sublattice of $M_{k}$ adsorption nodes at a given stage of the simulation. According to this, we defined $\Theta_{k}=\frac{N_{A g}^{k}}{M_{k}}$, where $N_{A g}^{k}$ is the number of $\mathrm{Ag}$ atoms adsorbed on kink sites. It can be clearly observed that none of the isotherms present an abrupt change in the coverage degree, showing a rather langmuirian shape that indicates that cooperative effects are absent in this type of adsorption. Another remarkable feature that is relevant for the discussion below is the fact that for increasing density of islands and the concomitant increasing roughness of their borders, the adsorption isotherms are shifted towards more negative chemical potentials.

It is clear that the kink sites subject of the present analysis are not all equivalent. Furthermore, many of them are actually not independent since the distance between them is lower than the cutoff radius for the interactions and they can even move in the course of the simulation due to the mobility allowed to the $\mathrm{Au}$ atoms. It may thus be interesting to compare the present isotherms for kink sites with others where the equivalence and independence of the adsorption nodes is granted a priori by considering an ideal ensemble of identical and independent sites. With this purpose we built such lattices with the most commonly found kink sites, resulting from our simulated annealing procedure. The most commonly found types of kink sites are shown in figure 8 . With the adsorption energies corresponding to each of these sites we constructed the corresponding Langmuir isotherms for the coverage degree $\Theta_{i}^{\operatorname{Lang}}$ as a function of the chemical potential $\mu$. These isotherms were then used to compose what we shall denominate an "ideal " adsorption isotherm for Ag adsorption on kink sites $\Theta_{k i n k}^{i d e a l} / \mu$ according to the following. Let us denote with $\rho_{k}^{1}, \rho_{k}^{2}$ and $\rho_{k}^{3}$ the densities of each of the types of kink sites calculated from the simulated 
annealing results according to:

$$
\rho_{k}^{i}=\frac{M_{k}^{i}}{M_{k}}
$$

with $M_{k}^{i}$ denoting the number of kink sites of type $i$ and $M_{k}=M_{k}^{1}+M_{k}^{2}+M_{k}^{3}$. From the three isotherms $\Theta_{1}^{\text {Lang }} / \mu, \Theta_{2}^{\text {Lang }} / \mu$ and $\Theta_{3}^{\text {Lang }} / \mu$, we defined the "ideal " adsorption isotherms for adsorption on kink sites as:

$$
\Theta_{\text {kink }}^{\text {ideal }}(\mu)=\rho_{k}^{1} \Theta_{1}^{\text {Lang }}+\rho_{k}^{2} \Theta_{2}^{\text {Lang }}+\rho_{k}^{3} \Theta_{3}^{\text {Lang }}
$$

Ideal kink sites isotherms for the five imperfect structures selected are shown in Fig. 7b. Comparison with Fig. 7a indicates that the more compact the islands the closer the behaviour of the real isotherms to the ideal ones. On the other hand, for increasing roughness of the island borders, the real isotherms shift their inflection point towards lower chemical potentials. This larger deviation from ideality in the case of the rougher structures is understandable, since in this case the distance between kink sites diminishes and they no longer behave as independent adsorption sites.

Table 3 summarizes the chemical potentials at the inflection point $\left(\mu_{\text {infl }}\right)$ for real and ideal isotherms. Comparison with the value for the deposition of a Ag monolayer on a perfect surface shows that the presence of point defects may shift the threshold for underpotential deposition in the negative direction as much as $0.2 \mathrm{~V}$.

It is also interesting to compare these values with that corresponding to the filling of $\mathrm{Au}$ surface monovacancies with $\mathrm{Ag}$ atoms. In this case we obtained $\mu_{\text {infl }}^{v a c}=-3.53 \mathrm{eV}$. However, these defects are expected to have a too low concentration at the working temperatures in electrochemistry so their relevance may be considered as negligible for practical purposes.

\subsubsection{Deposition on the border of monoatomic steps}

The second stage of the present electrodeposition process involves the formation of one-dimensional phases through the decoration of monoatomic steps. In all cases this process was found in the $\mu$ range between $-3.05 \mathrm{eV}$ and $-2.95 \mathrm{eV}$, that is, after deposition on point defects and before the formation of the 2-D phase. The corresponding simulations were run with the five systems selected above, that is, a $100 \times 100$ lattice partially covered with $\mathrm{Au}$ islands and the chemical potential was run between $-3.32 \mathrm{eV}$ and $-2.90 \mathrm{eV}$. Figure 9 shows the adsorption isotherms for the surface configurations selected. Analogously to the case of point defects, we defined here a coverage degree related to border sites, $\Theta_{\text {step }}$, referred to the total number of adsorption sites located at the border of a monoatomic step in a given configuration of the simulation. If these isotherms are compared with those of figure $7 \mathrm{a}$, it can be noticed that the roughness of the $\mathrm{Au}$ islands plays the opposite role concerning the stability of the Ag deposit. That is, the increasing roughness of the islands shifts the isotherms towards more positive chemical potentials. This fact may be in principle explained in terms of the considerations made in section 4.1 about the influence of $\mathrm{Au}$ atoms on the energy spectrum of $\mathrm{Ag}$ adsorption. Rougher islands provide adsorption sites with a lower coordination of the $\mathrm{Ag}$ border adatom with the $\mathrm{Au}$ atoms of the island, so that the adsorption energy of the adsorbates is in the average shifted 
towards more positive values. However, this is a rather simplified explanation and a more detailed study in terms of the surface configurations should be undertaken.

Finally, it is remarkable the fact that as the compactness and size of the islands increase, the isotherms become steeper, indicating some sort of cooperative effect in the formation of the 1-D phase.

\subsubsection{Formation of $\mathrm{Ag}$ monolayers in the presence of surface defects}

The formation of the 2-D phase in the presence of Au islands was finally investigated in connection with the other processes described above. Chemical potential sweeps were undertaken in the range $[-3.30 \mathrm{eV}<\mu<-2.90 \mathrm{eV}]$, with the observation of adsorption of $\mathrm{Ag}$ atoms on kink sites $[-3.30 \mathrm{eV}<\mu<$ $-3.02 \mathrm{eV}]$, decoration of monoatomic steps, $[-3.05 \mathrm{eV}<\mu<-2.95 \mathrm{eV}]$ and the final formation of the monolayer $[-2.95 \mathrm{eV}<\mu<-2.90 \mathrm{eV}]$. Figure 10 shows the final state at different $\mu$ for one of the five systems considered previously $(M C S=5120)$.

If we compare the isotherms for this system(Fig. 11) with those corresponding to adsorption on perfect surfaces(Fig 4a), it is clear that the presence of surface defects introduces additional stages in the deposition process due to the formation of 0-D and 1-D phases. It is remarkable the smoothing of the isotherms in the neighborhood of the deposition potential of the monolayer, this being enhanced for rougher substrates. It is also observed that as the number of islands per unit surface increases, the chemical potential for the formation of the monolayer is shifted negatively. In all cases the formation of the 2-D phase is built in a coalescence regime starting from the Au islands. This can be noted in the snapshots of Figure 10.

All the deposition stages described occur at chemical potentials negative with respect to $\mathrm{Ag}$ bulk deposition $\left(\mu_{A g / A g}^{c}=-2.865 \mathrm{eV}\right.$ ), thus within the underpotential deposition range. The underpotential shifts $\Delta \phi_{U P D}$ for the different processes thus range between $0.4 \mathrm{eV}$ and $0.03 \mathrm{eV}$.

\section{Conclusions}

The deposition of $\mathrm{Ag}$ on $\mathrm{Au}(100)$ was studied in the absence and in the presence of surface defects. These were generated by means of a simulated annealing procedure using different numbers of Monte Carlos steps (MCS) per cooling cycle. It was found that a lower number of $(M C S)$ generates a higher number of $\mathrm{Au}$ islands per unit surface, which are characterized by a higher perimeter/area rate. On the other hand, a lower cooling rate generates more compact, bigger $\mathrm{Au}$ islands.

$\mathrm{Ag}$ deposition always takes place at chemical potentials lower than bulk deposition, thus leading to a positive underpotential shift. This is also supported by first-principles calculations performed by one of us [36. The adsorption isotherms obtained indicate that $\mathrm{Ag}$ adsorption should occur in three well defined stages: first, adsorption on kink sites, second, adsorption at the border of monoatomic steps and finally formation of the monolayer or 2-D phase. In all cases the presence of inhomogeneities shifts the adsorption isotherms towards more negative chemical potentials thus increasing the underpotential shift with 
respect to that predicted for a perfect surface. Furthermore, the isotherms are smoothed in the neighbourhood of the critical chemical potential, this effect being more pronounced for stronger disordered surfaces.

The results of our simulations support to a large extent the experimental results obtained for this system, fundamentally concerning the existence of a positive underpotential shift and the strong affinity of $\mathrm{Ag}$ for $\mathrm{Au}$ evidenced in the trend to build surface alloys. More detailed experimental studies concerning the influence of surface defects on the voltammograms and adsorption isotherms would be very helpful to check the present calculations and to improve the modelling of the system $\mathrm{Ag} / \mathrm{Au}(100)$. One point to be considered is that of finite size effects of the simulation cell, especially in the case of adsorption on kink sites for imperfect surfaces obtained at a low cooling rate.

\section{Acknowledgments}

Part of the present calculations were performed on a Digital workstation donated by the Alexander von Humboldt Stiftung, Germany. Fellowships from the Consejo de Investigaciones Científicas y Técnicas de la Provincia de Córdoba(C.G. and M.D. P.), financial support from the Consejo Nacional de Investigaciones Científicas y Técnicas, the Secretaría de Ciencia y Técnica de la Universidad Nacional de Córdoba, the Consejo de Investigaciones Científicas y Técnicas de la Provincia de Córdoba and language assistance by Pompeya Falcón are gratefully acknowledged. 


\section{Tables}

\begin{tabular}{|c|c|c|}
\hline$R \backslash N \times N$ & $40 \times 40$ & $100 \times 100$ \\
\hline \hline 2 & -2.975 & -2.970 \\
\hline 3 & -2.925 & -2.936 \\
\hline
\end{tabular}

Table 1: Threshold chemical potentials for $\mathrm{Ag}$ deposition on $\mathrm{Au}(100)$ in absence of surface defects. Different lattice sizes $(N \times N)$, and cutoff radii $(R)$ for the interactions were employed.

\begin{tabular}{|c|c|c|c|c|c|c|c|c|}
\hline$M C S$ & 20 & 40 & 80 & 160 & 320 & 640 & 1280 & 2560 \\
\hline \hline$\rho_{k}$ & 0.0261 & 0.0235 & 0.0221 & 0.0197 & 0.0163 & 0.0157 & 0.0132 & 0.0117 \\
\hline$\rho_{s}$ & 0.108 & 0.1044 & 0.0991 & 0.0919 & 0.0877 & 0.0716 & 0.0694 & 0.0560 \\
\hline$\frac{P}{A}$ & 82.33 & 32.33 & 20.27 & 12.88 & 8.25 & 4.12 & 3.29 & 1.96 \\
\hline
\end{tabular}

\begin{tabular}{|c|c|c|c|c|c|c|c|c|}
\hline$M C S$ & 5120 & 10240 & 20480 & 40960 & 81920 & 163840 & 327680 & 655360 \\
\hline \hline$\rho_{k}$ & 0.0105 & 0.0081 & 0.0068 & 0.0064 & 0.0050 & 0.0029 & 0.0027 & 0.0030 \\
\hline$\rho_{s}$ & 0.0432 & 0.0392 & 0.0353 & 0.0260 & 0.0184 & 0.0172 & 0.0145 & 0.0142 \\
\hline$\frac{P}{A}$ & 1.26 & 0.94 & 0.77 & 0.56 & 0.35 & 0.27 & 0.23 & 0.24 \\
\hline
\end{tabular}

Table 2:Density of kink sites $\rho_{k}$, density of border sites at the monoatomic steps $\rho_{s}$ and perimeter to area relationship $\frac{P}{A}$ of the $\mathrm{Au}$ islands generated by simulated annealing. $M C S$ is the total number of Monte Carlo steps employed for the annealing process between $10.000 \mathrm{~K}$ and $300 \mathrm{~K}$.

\begin{tabular}{|c|c|c|c|c|c|}
\hline$M C S$ & 20 & 320 & 5.120 & 81.920 & 655.360 \\
\hline \hline$\mu_{\text {infl }}$ & -3.14 & -3.12 & -3.11 & -3.11 & -3.11 \\
\hline$\mu_{\text {infl }}^{\text {ideal }}$ & -3.12 & -3.11 & -3.11 & -3.11 & -3.10 \\
\hline
\end{tabular}

Table 3 Chemical potential $\mu_{i n f l}$ in $\mathrm{eV}$ corresponding to the isotherms resulting from the simulation of $\mathrm{Ag}$ deposition on kink sites. These belong to $\mathrm{Au}$ imperfect surfaces generated by simulated annealing with different number of Monte Carlos steps $(M C S)$. $\mu_{\text {infl }}^{\text {ideal }}$ are the corresponding values for the inflection points of the ideal isotherms. 


\section{Figure Captions}

Figure 1 a) Some of the most common surface defects found on single crystal surfaces: 1) flat terrace, 2) monoatomic step, 3) kink site. b), c), d) different steps of metal deposition.

Figure 2 Environment of $\mathrm{S}$ sites ( a) $\mathrm{S}=9$, b) $\mathrm{S}=13$ ) surrounding an adsorption node. These were employed for the calculation of the adsorption/desorption energies of $\mathrm{Ag}$ and $\mathrm{Au}$ on $\mathrm{Au}(100)$ for a cutoff radii corresponding to the distance between second (a) and third (b) nearest neighbours. The particle adsorbed is located in the central box (9 and 13 respectively) .

Figure 3 Adsorption energies of a $\mathrm{Ag}$ atom at the center of the environment shown in figure $2 \mathrm{~b}$. The histogram drawn with the continuous line considers only the configurations involving $\mathrm{Ag}$ atoms, while those configurations involving only $\mathrm{Au}$ atoms were drawn with the dotted line. The plot drawn with pulses includes all the possible configurations that may be obtained with the environment including 13 adsorption nodes. In all cases the proper normalizing factors were employed( 4096, 4096 and 531441 respectively).

Figure 4 a) Adsorption isotherms for $\mathrm{Ag}$ deposition on defect free $\mathrm{Au}(100)$ (1-4) and $\operatorname{Ag}(100)$ (5) single crystal surfaces. Different lattice sizes $(N \times N)$ and cutoff radii $R$ were employed. In each case $R=n$ denotes that the distance to the $n^{\text {th }}$ nearest neighbour was employed. 1) $N=100, R=3$; 2) $N=40, R=2$; 3) $N=40, R=3$; 4) $N=100, R=2$; 5) $N=40$ and $R=3$.

b) Hysteresis effects for the simulation of $\mathrm{Ag}$ adsorption on $\mathrm{Au}(100)$. The simulations were performed on a $100 \times 100$ lattice. The cutoff radius corresponded to $R=3$ and the starting conditions for the coverage degree were 1) $\Theta_{A g}=1$; 2) $\Theta_{A g}=0.5$ and 3) $\Theta_{A g}=0$

Figure 5 Adsorption isotherms for $\mathrm{Ag}$ adsorption on $\mathrm{Au}(100)$ at different temperatures. .

Figure 6 Final configurations for the simulated annealing simulations. The number of Monte Carlo steps $N_{M C S}$ increases from upper left to down right. $N_{M C S}=20 \times 2^{m-1}$, where $m$ is the ordinal number of the configuration in the figure.

Figure 7 a)Adsorption isotherms for Ag deposition on kink sites for different types of imperfect surfaces obtained through the simulated annealing procedure.

b)Ideal isotherms calculated according to the procedure indicated in the text for the different types of imperfect surfaces.

Figure 8 Types of kink sites most commonly found after the simulated annealing procedure.

Figure 9 Adsorption isotherms for decoration of monoatomic steps. The $\mathrm{Au}$ surface structures considered were the final states of simulated annealing runs with 20,320,5120, 81920 and 655360 MCS per temperature cycle respectively.

Figure 10 Final state at different chemical potentials for a system in which the Au islands were obtained by a simulated annealing process with 5120 MCS per cooling cycle. The Au atoms on the surface are represented by black pixels and the $\mathrm{Ag}$ atoms by grey pixels. 1) $\mu=-3.29 \mathrm{eV}, 2) \mu=-3.07 \mathrm{eV}, 3$ ) $\mu=-2.97 \mathrm{eV}, 4) \mu=-2.94 \mathrm{eV}, 5) \mu=-2.93 \mathrm{eV}, 6) \mu=-2.91 \mathrm{eV}$ 
Figure 11 Adsorption isotherms for $\mathrm{Ag}$ deposition on $\mathrm{Au}(100)$ in the presence of surface defects. Each curve corresponds to one of the five systems considered previously. 


\section{References}

[1] E. Budevski, G. Staikov and W. J. Lorenz, Electrochemical Phase Formation and Growth, VCH Weinheim, 1996.

[2] E. P. M. Leiva, in "Current Topics in Electrochemistry", Vol. 2, pag. 269, Council of Scientific Information, Trivandrum, India,(1993).

[3] E. P. M. Leiva, Electrochim. Acta 41, 2185, (1996).

[4] D.M. Kolb, M. Przasnyski and H. Gerischer, J. Electroanal. Chem.

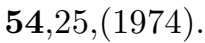

[5] S. Trasatti, Zeits. für Phys. Chem. NF , 98,75 (1975).

[6] K. Jutttner, G. Staikov, W.J. Lorenz, E. Schmidt, J. Electroanal. Chem. 80, 67 (1977).

[7] A. Bewick, B. Thomas, J. Electroanal. Chem. 65, 911 (1975).

[8] A. Bewick, B. Thomas, J. Electroanal. Chem. 70, 239 (1976).

[9] L. Blum, D. A. Huckaby and M. Legault, Electrochim. Acta 41, 2201 (1996).

[10] S. G. García, PhD. Thesis, Universidad Nacional del Sur, Bahía Blanca, Argentina, 1997.

[11] D. Carnal, P. I. Oden, U. Muller, E. Schmidt and H. Sigentaler, Electrochim. Acta 40, 1223 (1995).

[12] G. Staikov, K. Jutttner, W.J. Lorenz and E. Budevski, Electrochim Acta 23,319 (1978).

[13] M. F. Toney, J. G. Gordon, M. G. Sammant, G. L. Borges, O. R. Merloy , D. Yee and L. B. Sorensen, J. Phys. Chem. 9 9, 4733 (1995).

[14] M.G. Del Pópolo, E.P.M. Leiva, J. Electroanal. Chem. 440, 271 (1997).

[15] X. K. Xing, I.T. Bae and D. Scherson, Electrichimica Acta, bf 40, 29 (1995).

[16] C. G. Sánchez, M. G. Del Pópolo and E.P.M. Leiva, Surface Science, in press.

[17] Zhichao Shi, Shijie Wu and J. Lipkowski, Electrochimica Acta, 40 ,9 (1995).

[18] S. G. García, D. Salinas, C. Mayer, J.R. Vilche, H. J. Pauling, S. Vinzelberg, G. Staikov, W. J. Lorenz, Surface Science bf 316, 143 (1994).

[19] S. G. García, D. Salinas, C. Mayer, E. Schmidt, G. Staikov, W. J. Lorenz, Electrochim. Acta 43, 3007 (1998).

[20] N. Ikemiya, K. Yamada, S. Hara, Surface Science 348, 253 (1996). 
[21] D. Nicholson and N. G. Personage, Computer Simulation and Statistical Machanics of Adsorption, Academic Press, (1982).

[22] F. Celestini, D. Passerone, F. Ercolessi and E. Tosatti, Preprint SISSA $133 / 97 / \mathrm{CM} / \mathrm{SS}$.

[23] M. G. Samant, M. F. Toney, G. L. Borges, L. Blum and O. R. Melroy, J. Phys. Chem,92, 220 (1998).

[24] M.C. Gimenez, M. G. Del Pópolo and E.P.M. Leiva. "Portucalensis", Conference on Electrified Interfaces, Porto, Portugal, Julio de 1998.

[25] W. Schmickler and U. Stimming, J. Electroanal. Chem. 366 , 203 (1994).

[26] Carlsson, Solid State Physics, 43, H. Ehrenreich, H. Seitz, and D. Turnbull, p. 1, Academic Press, New York, (1990).

[27] S. M. Foiles, M. I. Baskes and M. S. Daw, Phys. Rev. B 337983 (1986).

[28] M. W. Finnis and J. E. Sinclair, Philos. Mag. A 50, 45 (1984).

[29] F. Ercolessi, E. Tosatti and M. Parrinello, Phys. Rev. Lett. 57, 719 (1986).

[30] C.L. Liu, J. M. Cohen, J.B. Adams and A. F. Voter. Surface Science 253, 334 (1991).

[31] M. I. Haftel, Phys. Rev. B, 48, 2611 (1993).

[32] M. P. Allen and D. J. Tildesley, Computer Simulation of Liquids, Oxford University Press, 1987.

[33] G. Brown, P. A. Rickvold, M. A. Novotny and A. Wieckowski, Colloid and Surfaces A, in Press, or cond-mat/9703209.

[34] P. Ruggerone, C. Ratsch and M. Scheffler, Growth of Ultrathin Epitaxial Layers, Vol. 8, Eds. D. A. King and D. P. Woodruff, Elsevier Science, Amsterdam, 1997.

[35] Dieter M. Kolb, in "Structurre of electrified interfaces" (Edited by J. Lipkowski, P. M. Ross), VCH, 1993

[36] C. G. Sánchez and E.P.M. Leiva, J. Electroanal. Chem. , in press. 


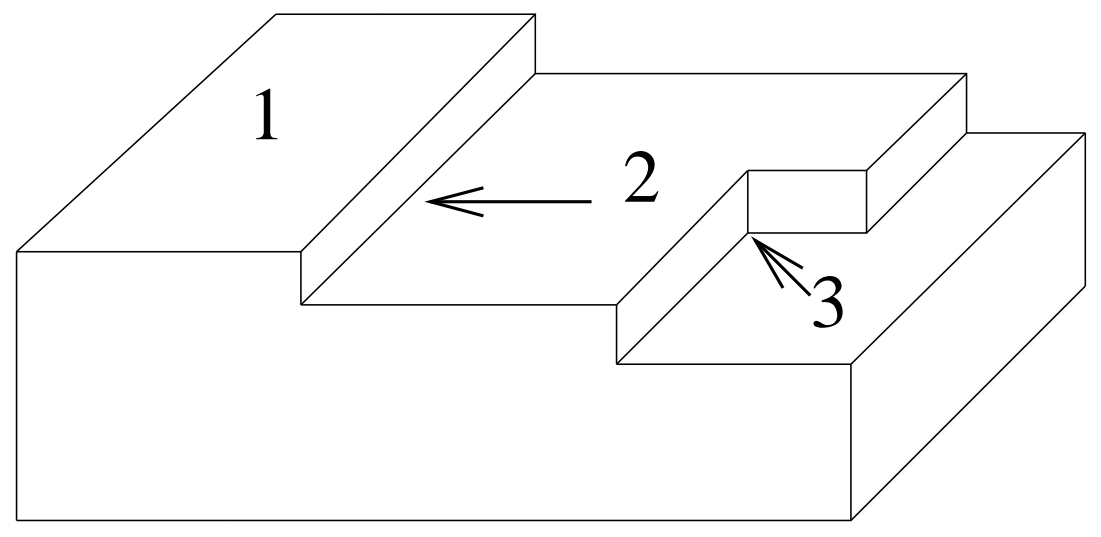

a

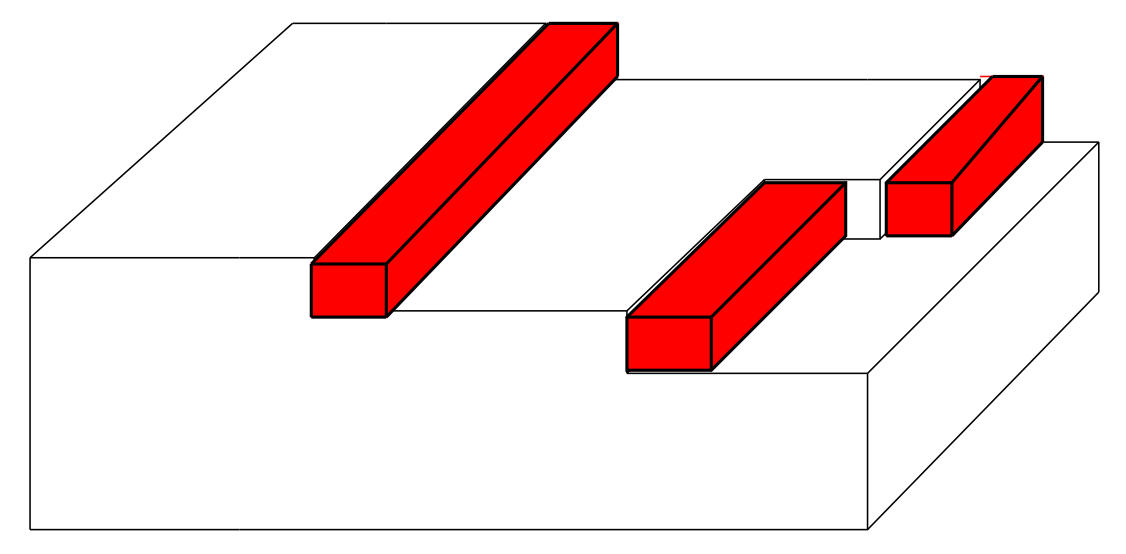

c

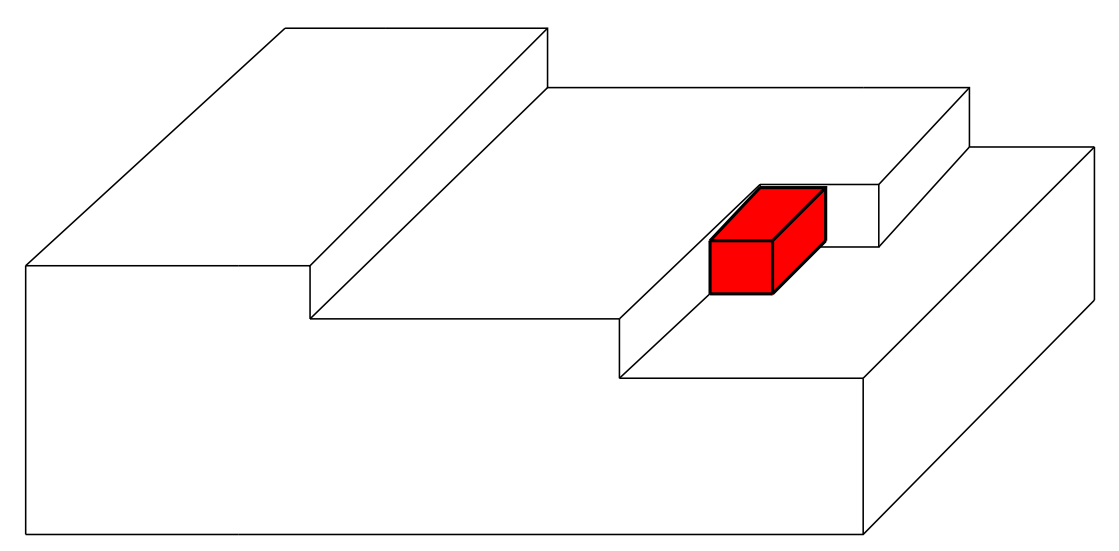

b

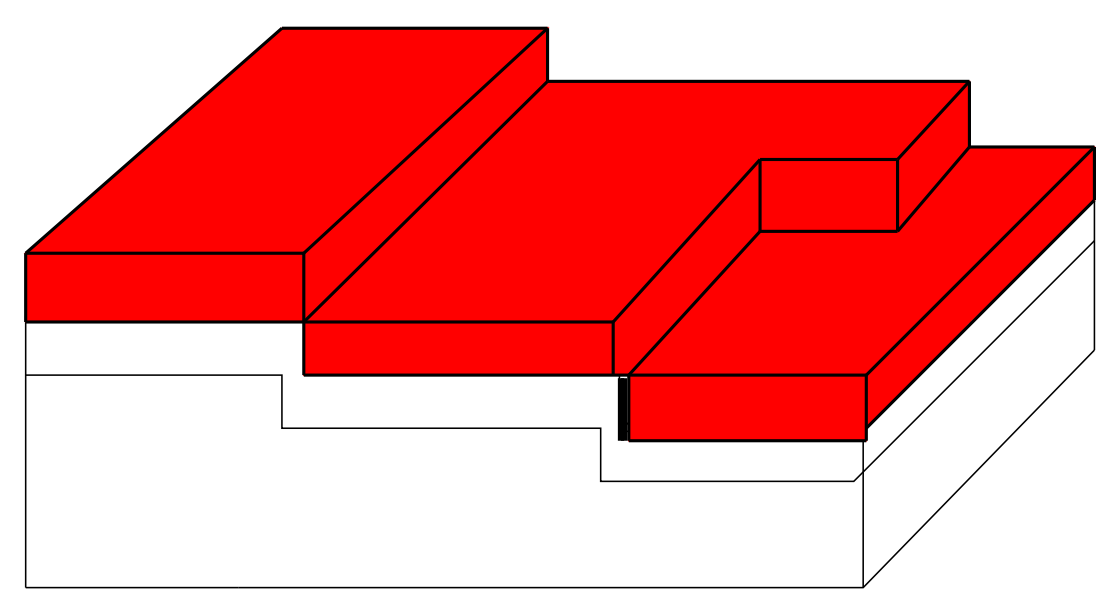

d 

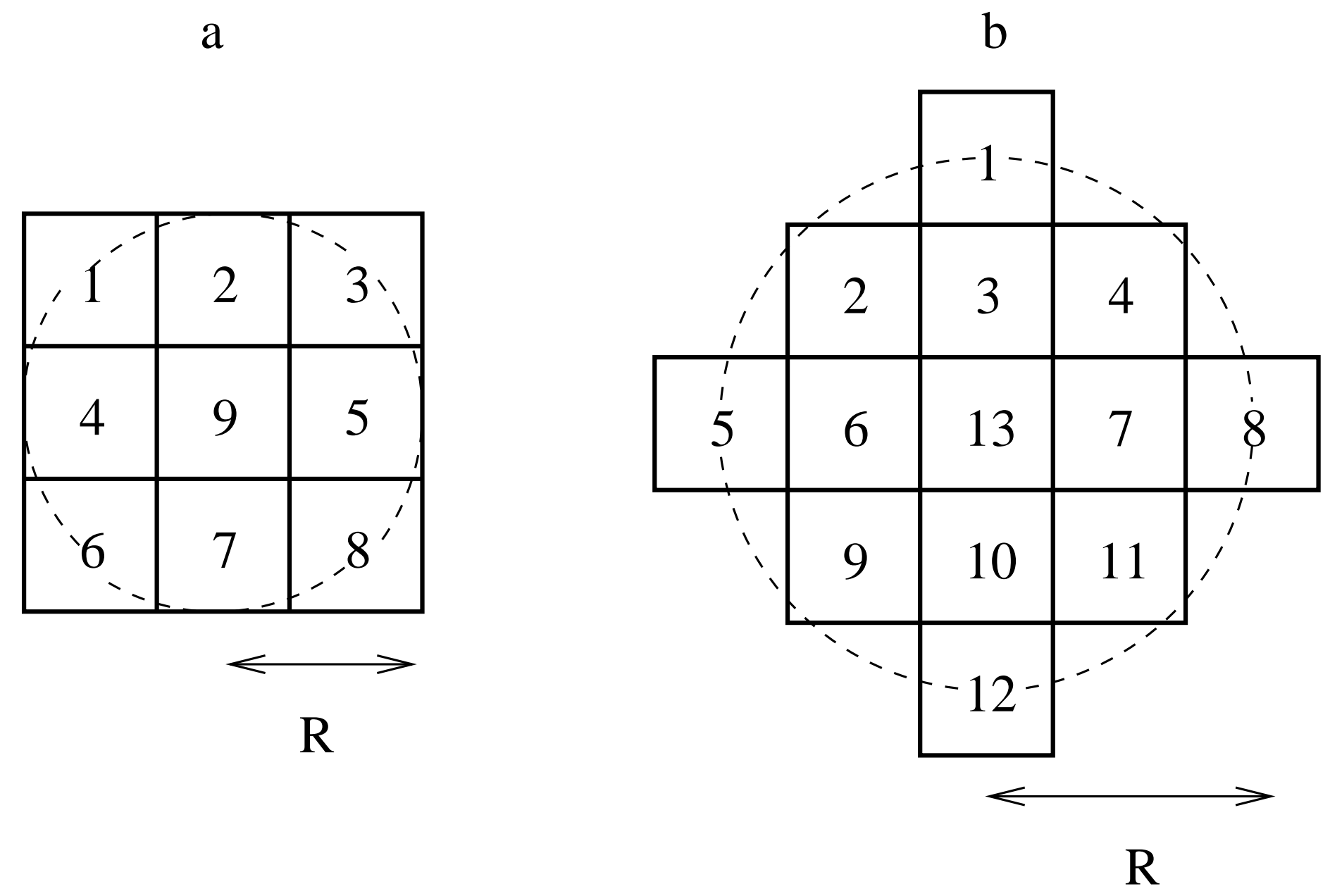


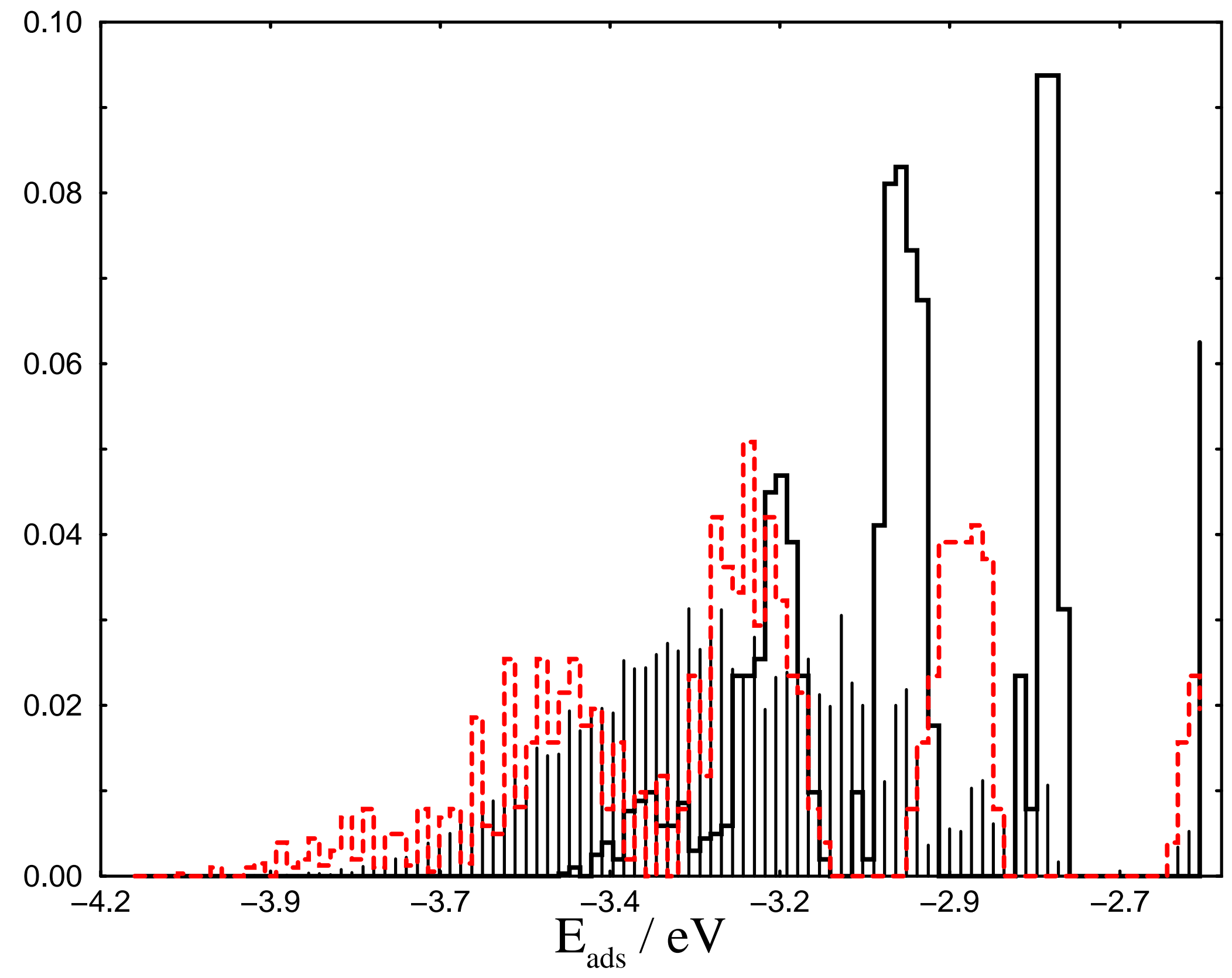




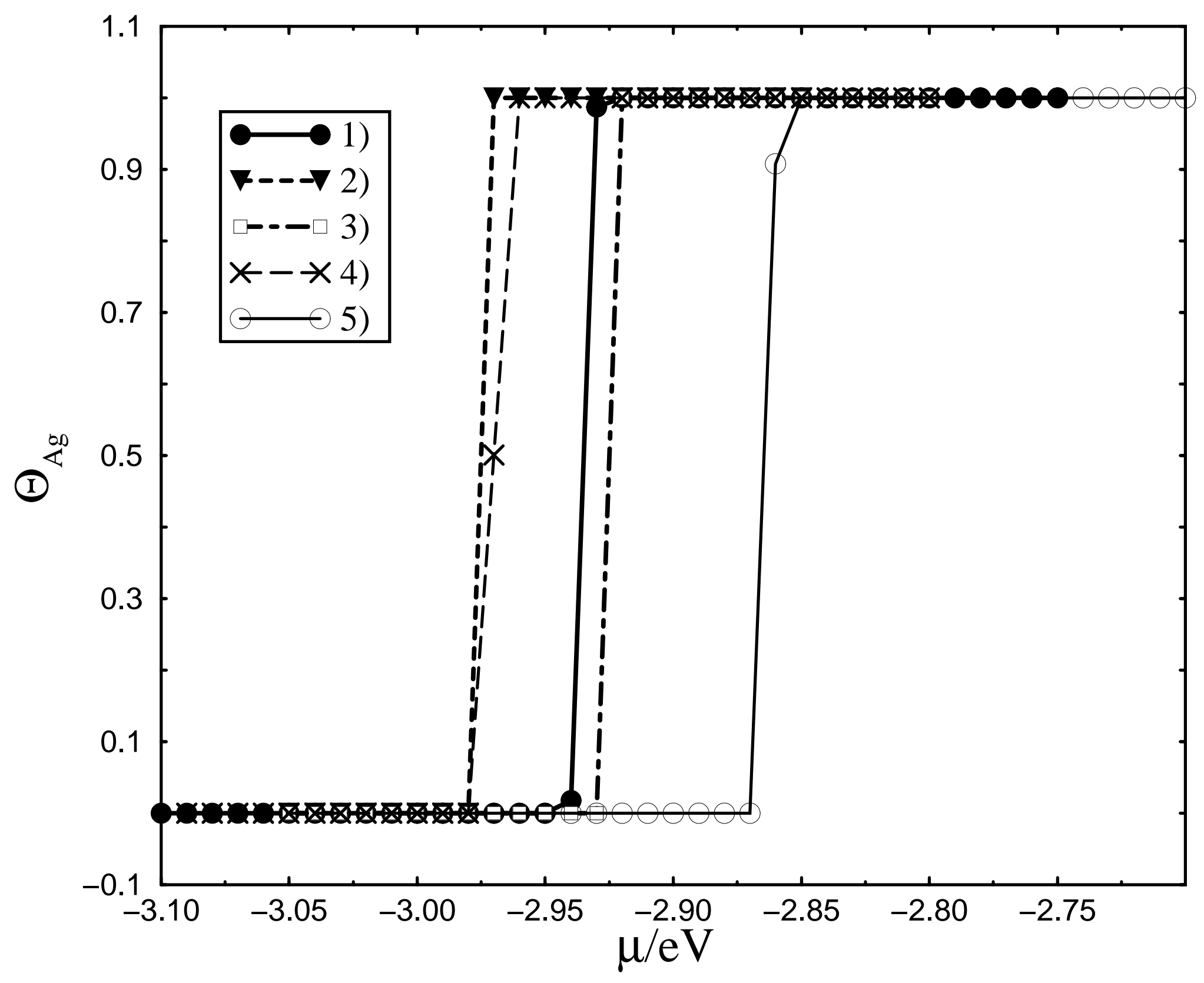




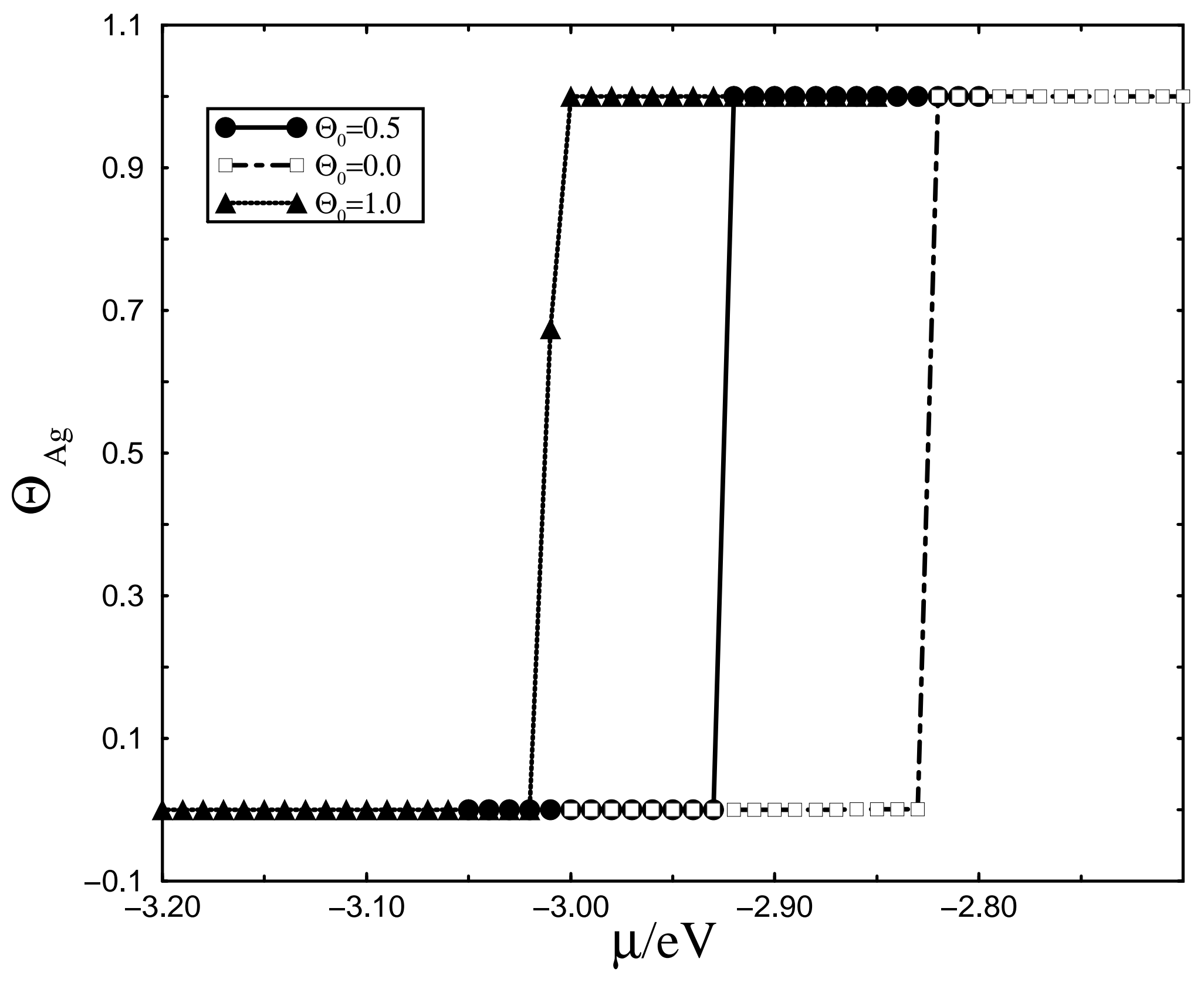




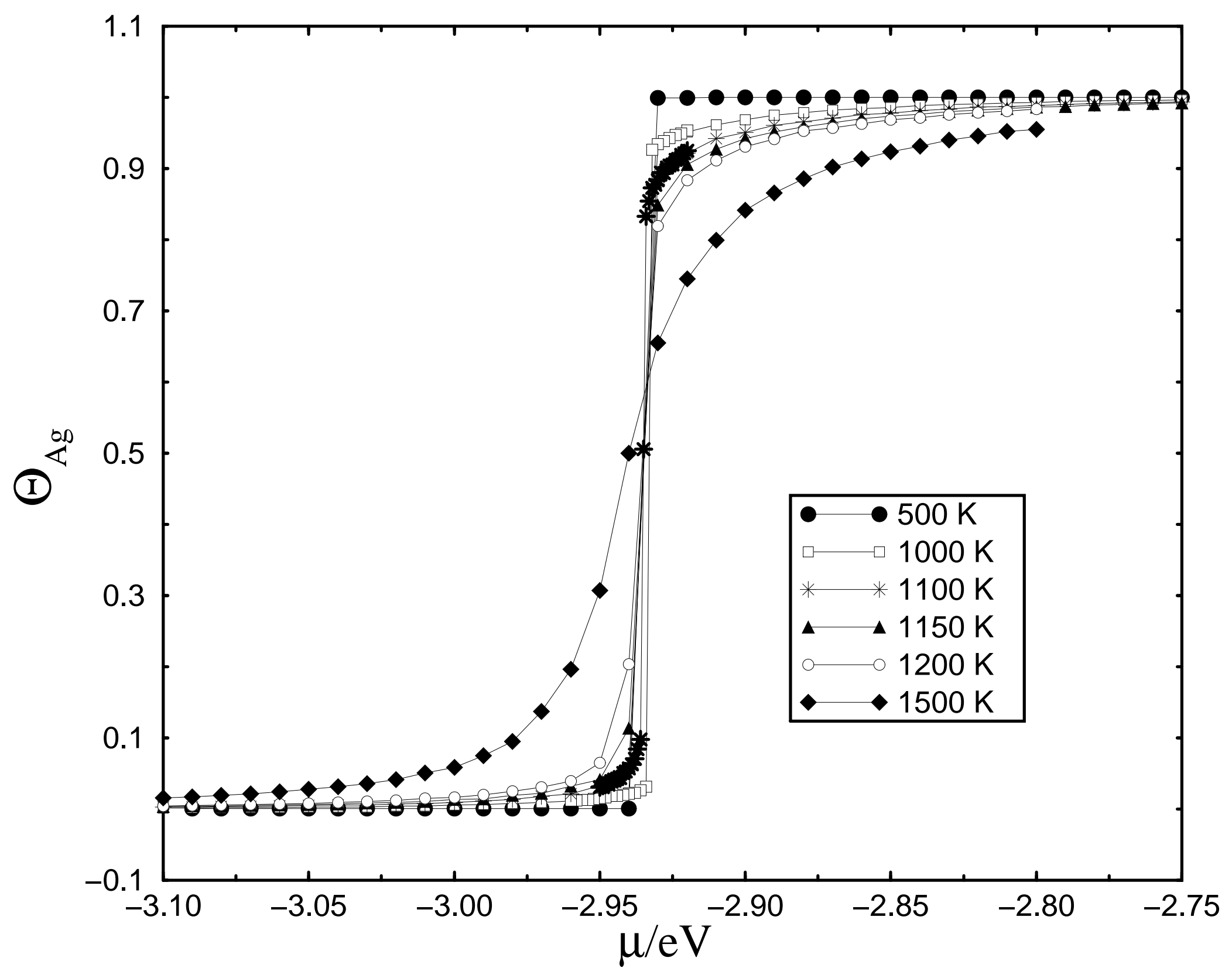




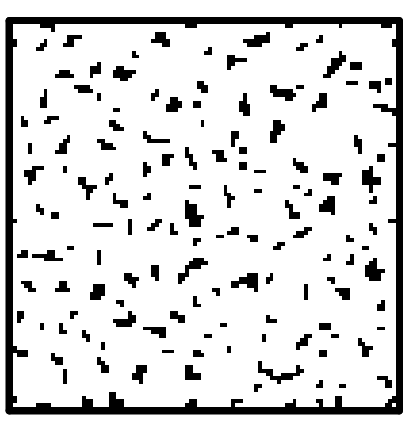

$$
1
$$

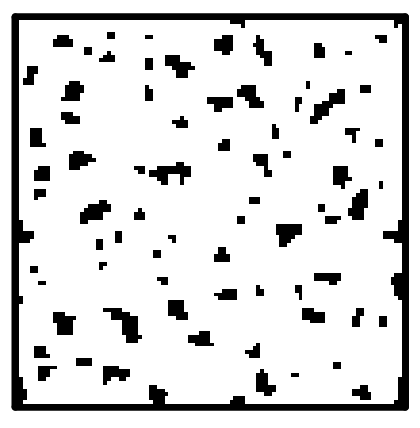

5

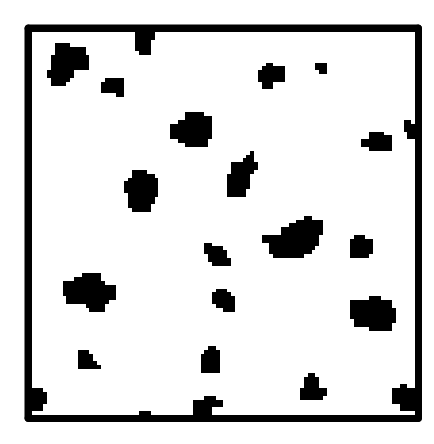

9

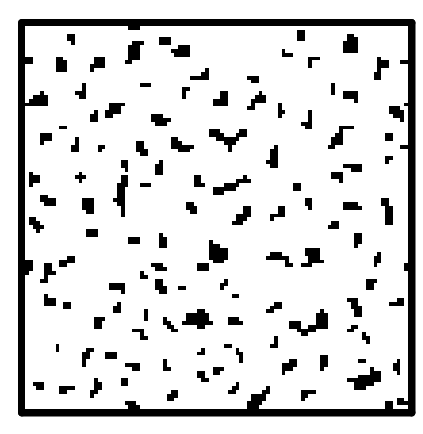

2

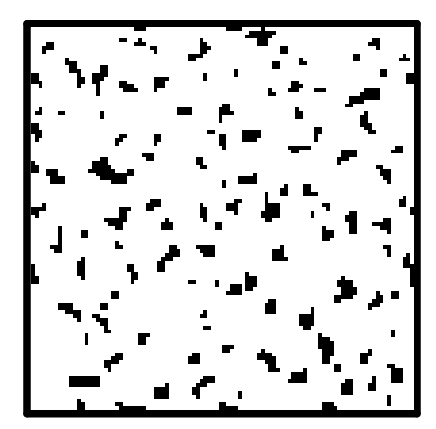

3

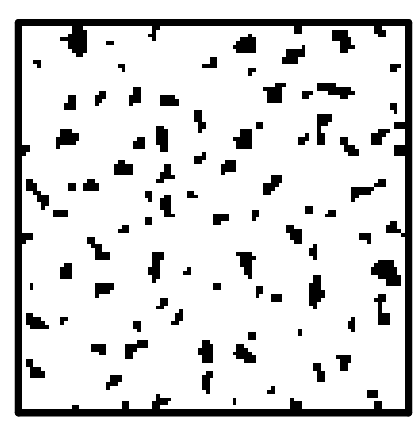

4

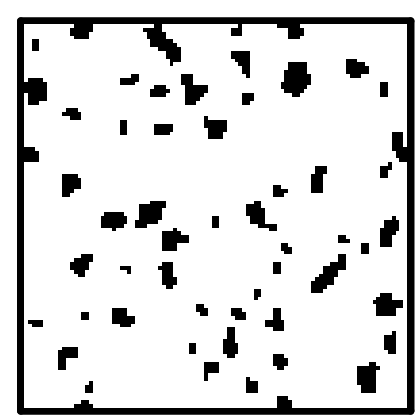

6

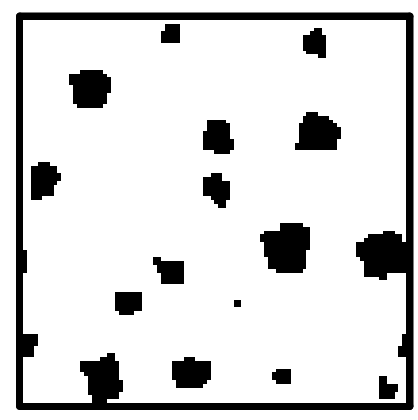

10

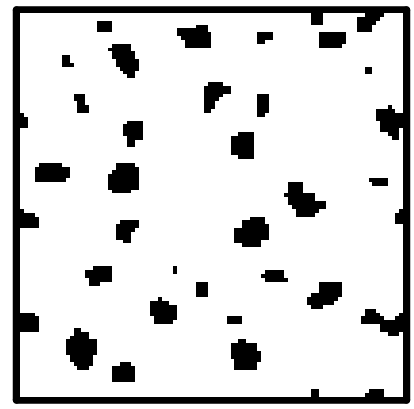

8

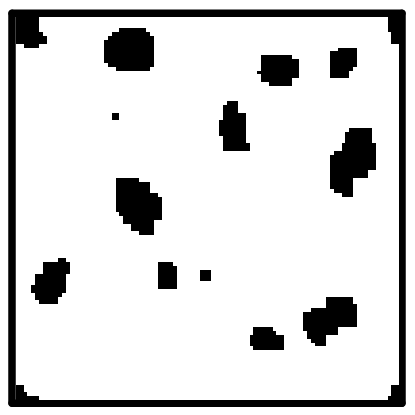

11

12

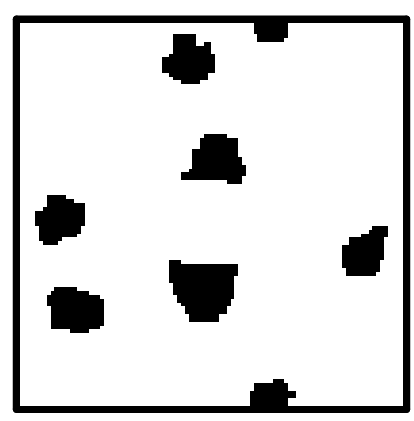

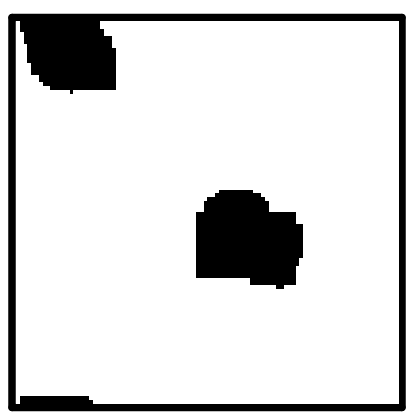

15

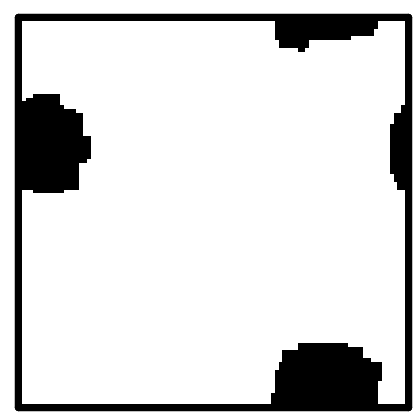

16 


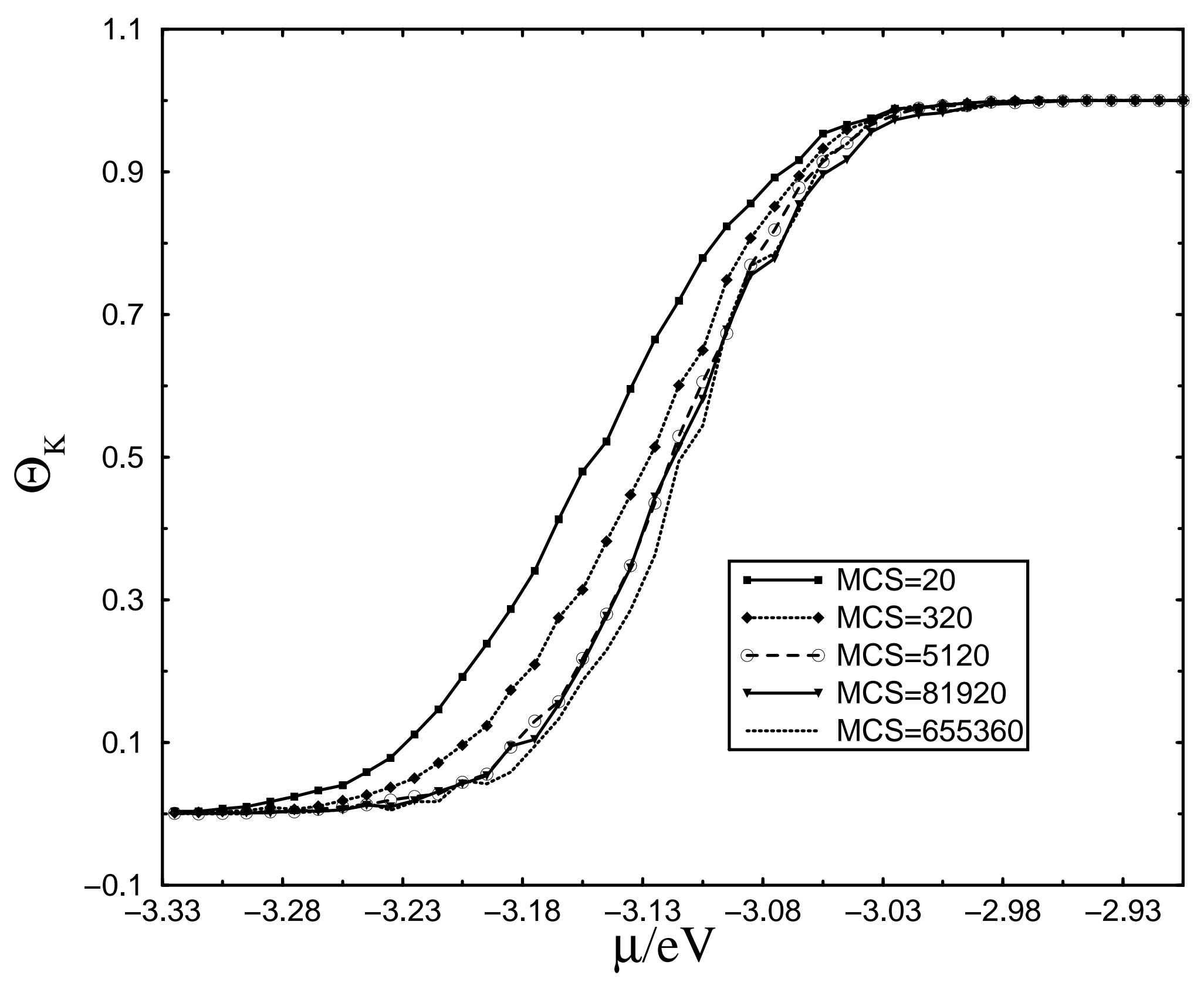




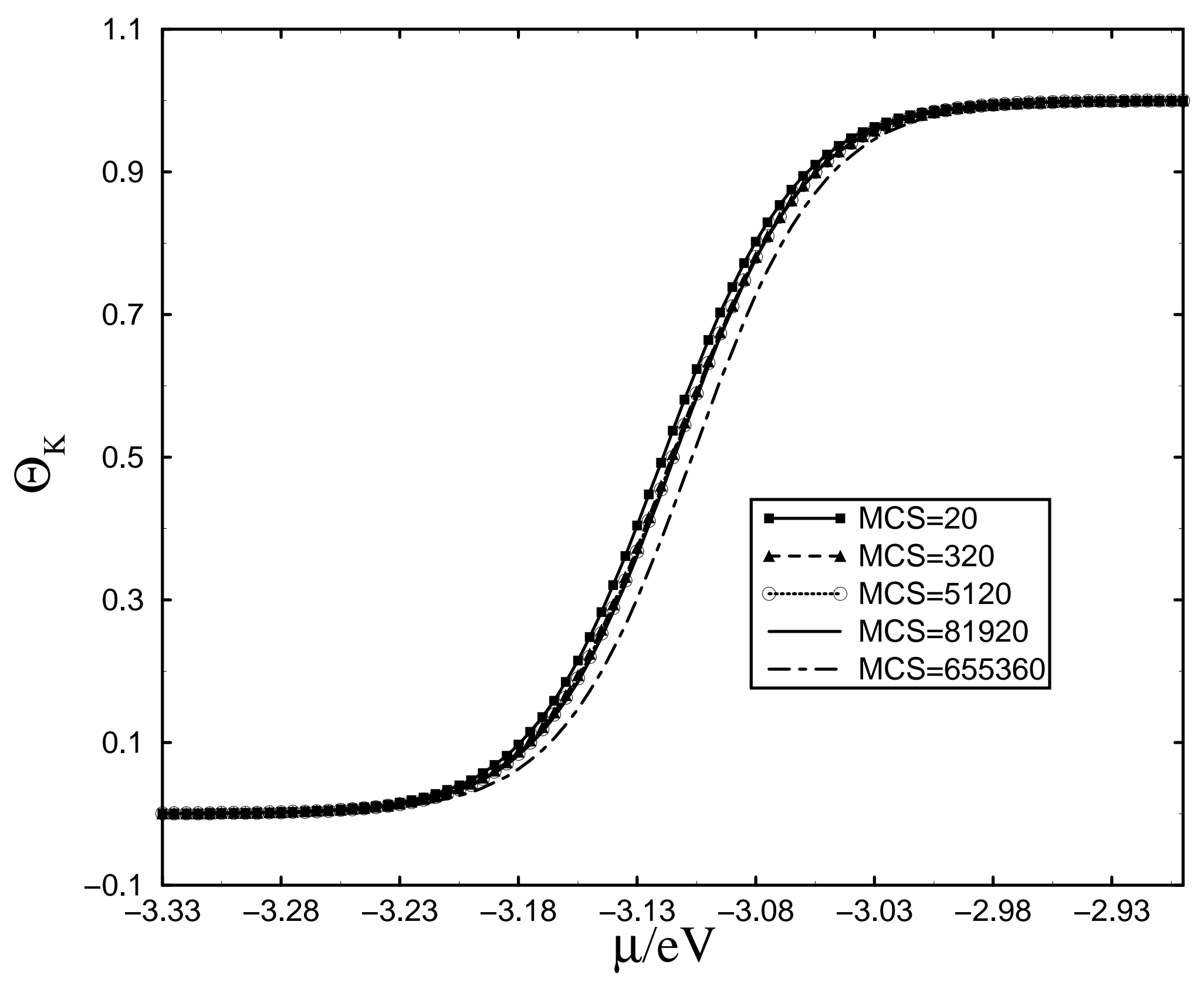


$\mathrm{K}=1$

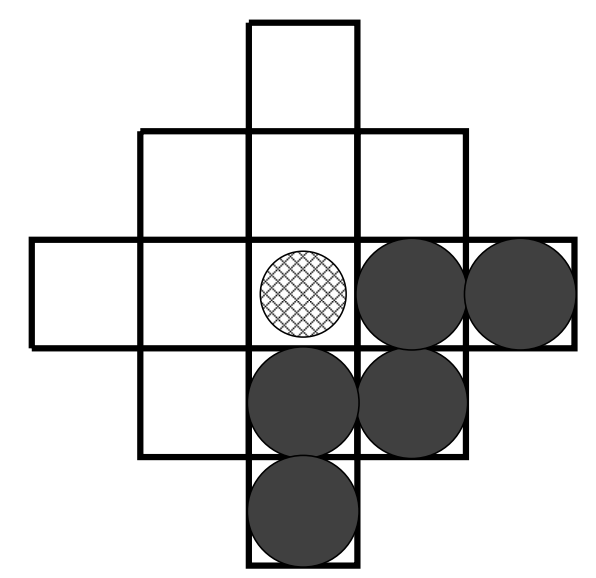

Eads $=-3.132 \mathrm{eV}$
$\mathrm{K}=2$

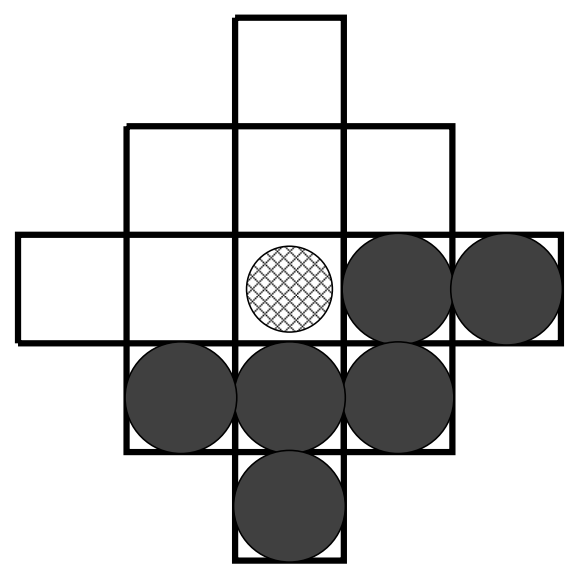

Eads $=-3.108 \mathrm{eV}$
$\mathrm{K}=3$

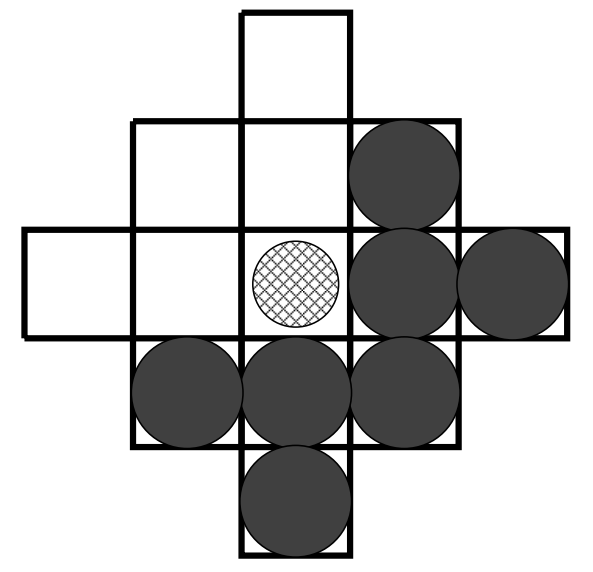

Eads $=-3.084 \mathrm{eV}$

Gold

Silver 


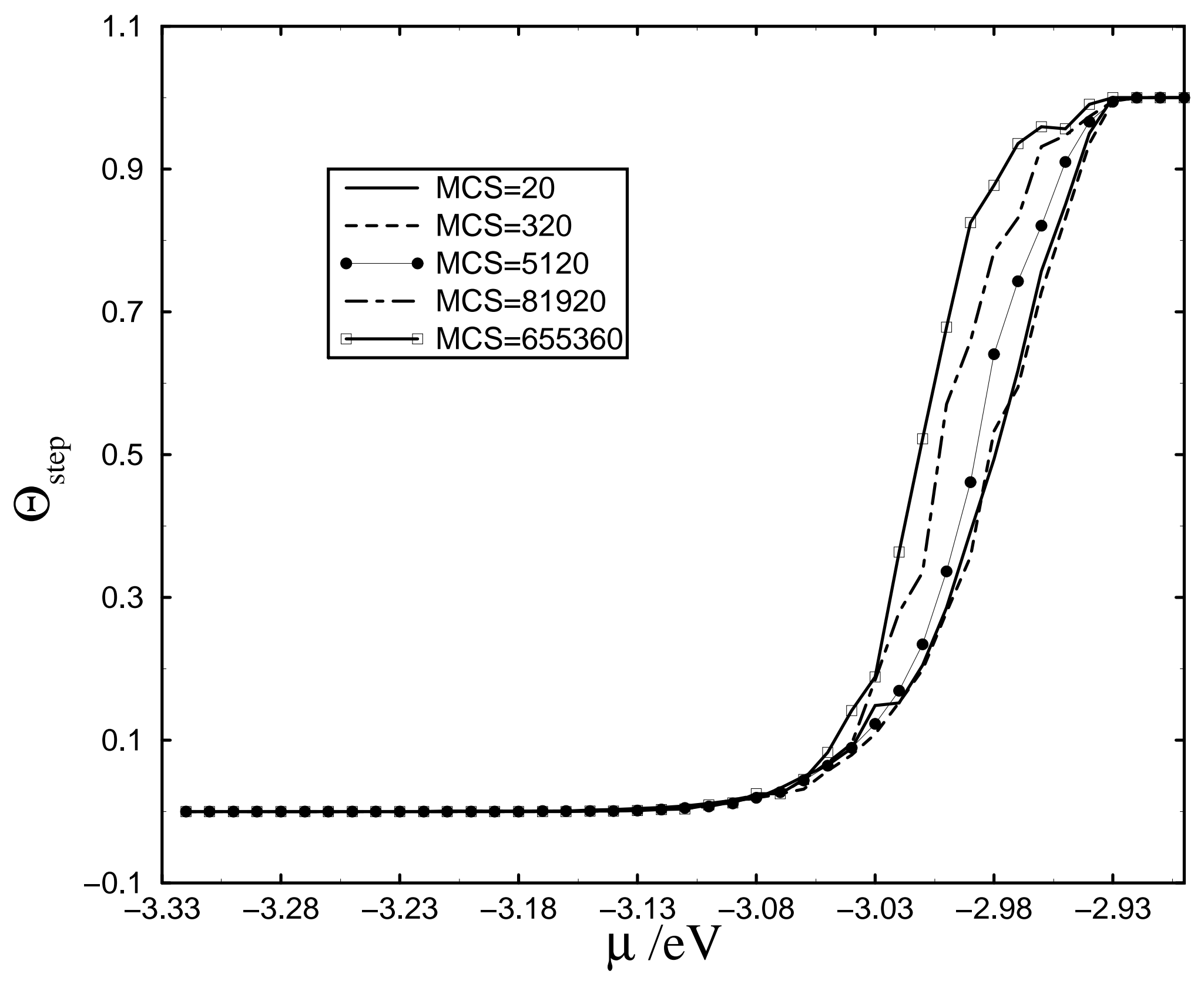



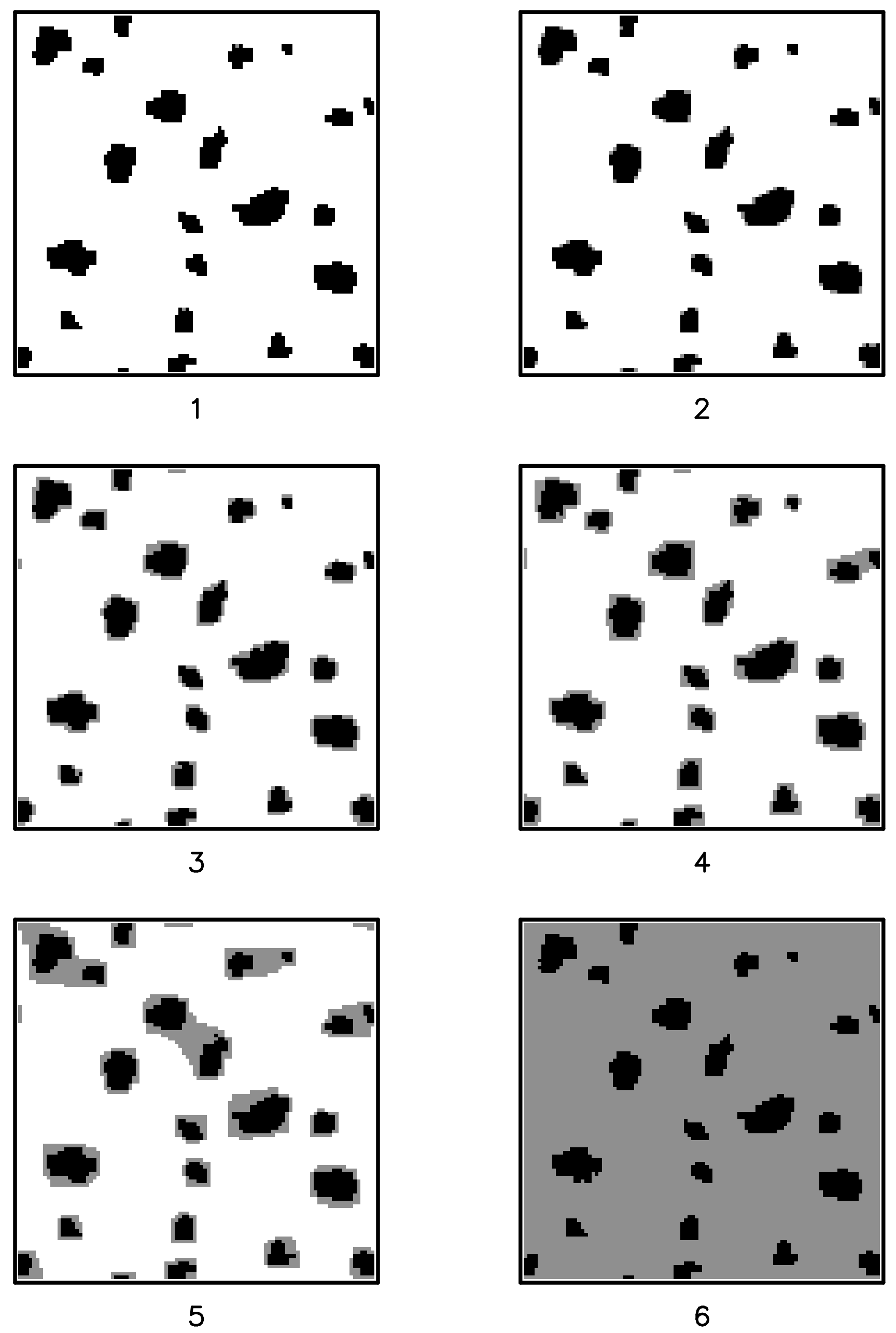


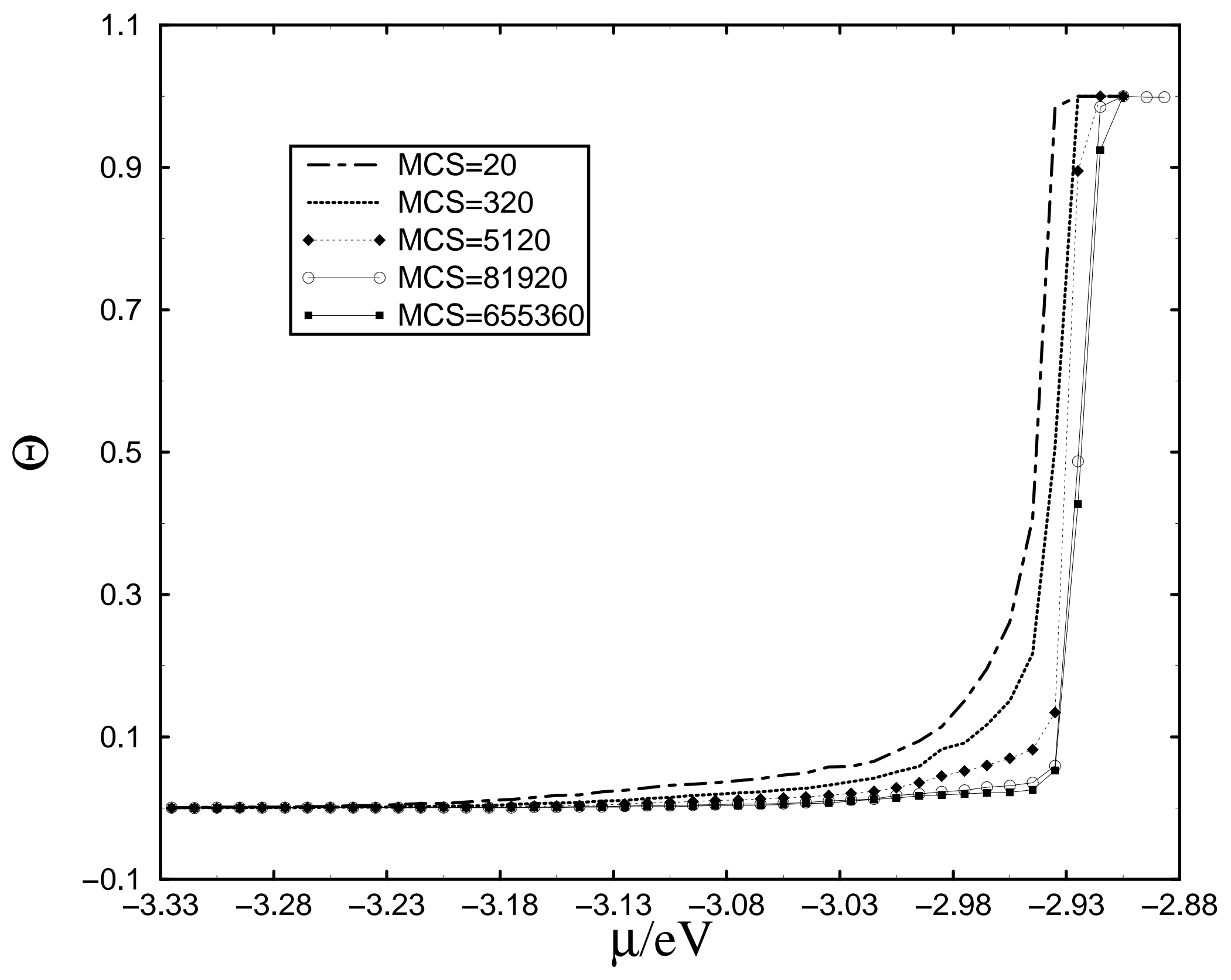

